ARTICLE

https://doi.org/10.1038/s41467-019-09356-x

\title{
Coordination of capsule assembly and cell wall biosynthesis in Staphylococcus aureus
}

\author{
Marvin Rausch (1) 1,2, Julia P. Deisinger ${ }^{1,2}$, Hannah Ulm¹, Anna Müller (1D 1, Wenjin Li ${ }^{3}$, Patrick Hardt', \\ Xiaogang Wang ${ }^{4}$, Xue Li ${ }^{4}$, Marc Sylvester (1) ${ }^{5}$, Marianne Engeser (1) ${ }^{6}$, Waldemar Vollmer ${ }^{7}$, Christa E. Müller (i) ${ }^{3}$, \\ Hans Georg Sahl ${ }^{8}$, Jean Claire Lee ${ }^{4} \&$ Tanja Schneider ${ }^{1,2}$
}

The Gram-positive cell wall consists of peptidoglycan functionalized with anionic glycopolymers, such as wall teichoic acid and capsular polysaccharide (CP). How the different cell wall polymers are assembled in a coordinated fashion is not fully understood. Here, we reconstitute Staphylococcus aureus $\mathrm{CP}$ biosynthesis and elucidate its interplay with the cell wall biosynthetic machinery. We show that the CapAB tyrosine kinase complex controls multiple enzymatic checkpoints through reversible phosphorylation to modulate the consumption of essential precursors that are also used in peptidoglycan biosynthesis. In addition, the CapA1 activator protein interacts with and cleaves lipid-linked CP precursors, releasing the essential lipid carrier undecaprenyl-phosphate. We further provide biochemical evidence that the subsequent attachment of CP is achieved by LcpC, a member of the LytR-CpsA-Psr protein family, using the peptidoglycan precursor native lipid II as acceptor substrate. The Ser/Thr kinase PknB, which can sense cellular lipid II levels, negatively controls CP synthesis. Our work sheds light on the integration of $\mathrm{CP}$ biosynthesis into the multi-component Gram-positive cell wall.

\footnotetext{
${ }^{1}$ Pharmaceutical Microbiology, University of Bonn, Bonn 53115, Germany. ${ }^{2}$ German Center for Infection Research (DZIF), partner site Bonn-Cologne, Bonn 53115, Germany. ${ }^{3}$ Pharma Center Bonn, Pharmaceutical Institute, Pharmaceutical Chemistry I, University of Bonn, Bonn 53121, Germany. ${ }^{4}$ Division of Infectious Diseases, Department of Medicine, Brigham and Women's Hospital, Harvard Medical School, Boston 02115 MA, USA. ${ }^{5}$ Institute of Biochemistry and Molecular Biology, University of Bonn, Bonn 53115, Germany. ${ }^{6}$ Kekulé Institute for Organic Chemistry and Biochemistry, University of Bonn, 53121 Bonn, Germany. ${ }^{7}$ Center for Bacterial Cell Biology, Medical School, Newcastle University, Newcastle upon Tyne NE2 4AX, UK. ${ }^{8}$ Institute of Medical Microbiology, Immunology and Parasitology, University of Bonn, Bonn 53127, Germany. These authors contributed equally: Marvin Rausch, Julia P. Deisinger, Hannah Ulm. Correspondence and requests for materials should be addressed to T.S. (email: tschneider@uni-bonn.de)
} 
T he bacterial cell envelope is a complex multilayered structure consisting of peptidoglycan (PG), which in Gram-positive bacteria is densely decorated with glycopolymers such as wall teichoic acid (WTA) and capsular polysaccharide (CP). The coordinated synthesis and assembly of these polymers is pivotal for maintenance of cell wall architecture and function ${ }^{1}$. In contrast to the biosyntheses of PG and WTA, for which the individual enzymatic reactions have already been characterized in vitro ${ }^{2,3}$, the biochemistry underlying capsule formation in Gram-positive bacteria is not well understood. Even more so, it is largely unknown how the different cell wall synthesis pathways, which share building blocks and membrane carriers, function in a coordinated and integrated fashion.

In the case of Staphylococcus aureus, an important opportunistic pathogen ${ }^{4}$, the expression of a polysaccharide capsule contributes substantially to the ability to cause invasive disease ${ }^{5-7}$. Serotype 5 and 8 capsular polysaccharide (CP5 and CP8) types are dominant among clinical isolates ${ }^{6}$. S. aureus USA300, which is prevalent in the United States, lacks a capsule due to several conserved mutations within the cap5 locus $^{8}$. However, the majority of USA300-associated infections involved superficial wounds or abscesses ${ }^{9}$, and USA300 isolates are not common outside of North America ${ }^{10}$. Among predominant methicillinresistant $S$. aureus clones worldwide are the CP8 + lineages ST1, ST30, ST59, ST80, and ST239 and the CP5 + lineages ST5 and ST22.
CP5 and CP8 share similar trisaccharide repeating units, which are identical in monosaccharide composition and sequence, only differing in the glycosidic linkages between the sugars and the sites of $O$-acetylation ${ }^{11}$.

The CP5 biosynthetic gene cluster comprises 16 genes (cap5Acap5P; Fig. 1a) ${ }^{12}$ encoding for proteins involved in polymer biosynthesis $7,13,14$, acetylation ${ }^{15}$, transport, and the regulation of CP production ${ }^{16,17}$. Database homology searches with amino acid sequences of cap5 operon gene products allowed for the prediction of individual enzymatic functions and the proposal of a pathway for capsule (CP) biosynthesis in S. aureus ${ }^{18}$.

Within this pathway (Fig. 1b), synthesis of the soluble building blocks occurs in the cytoplasm via three distinct reaction cascades, through which the universal cell envelope substrate UDP-D- $N$-acetylglucosamine (UDP-D-GlcNAc), is converted into the three different nucleotide-coupled sugars UDP- $N$-acetylD-fucosamine (UDP-D-FucNAc), UDP- $N$-acetyl-L-fucosamine (UDP-L-FucNAc) and UDP- $N$-acetyl-D-mannosaminuronic acid (UDP-D-ManNAcA). The synthesis of the first soluble precursor UDP-D-FucNAc is allegedly catalyzed in a two-step process by the enzymes CapD and CapN. Only recently, the integral membrane protein CapD was shown to function as a 4,6-dehydratase, which generates the intermediate UDP-2-acetamido-2,6-dideoxyD-xylo-4-hexulose ${ }^{19}$, proposed to be further converted to UDPD-FucNAc by the action of the membrane-associated reductase CapN, though experimental evidence is lacking so far. Subsequently, CapM is supposed to transfer the phosphosugar moiety
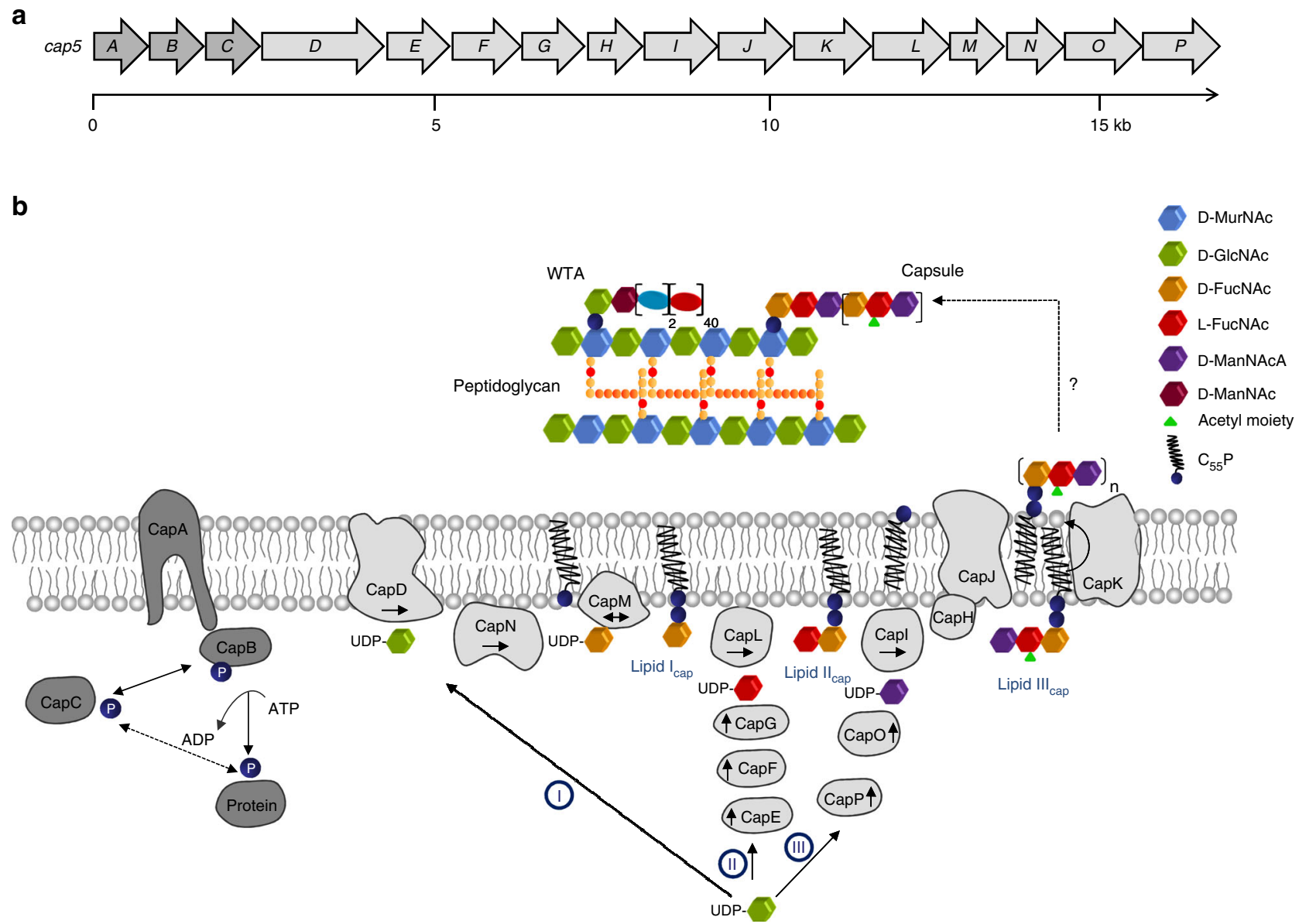

Fig. 1 S. aureus capsule biosynthesis. a S. aureus capsule (CP5) biosynthesis gene cluster (NWMN_0095-0110). b Model for the capsule (CP) biosynthesis pathway in $\mathrm{S}$. aureus and its regulation by the tyrosine kinase complex CapA1B1. $\mathrm{C}_{55} \mathrm{P}$, undecaprenyl-phosphate; GlcNAc, $\mathrm{N}$-acetyl-glucosamine; FucNAc, $\mathrm{N}$-acetyl-fucosamine; ManNAc, $\mathrm{N}$-acetyl-mannosamine; ManNAcA, $\mathrm{N}$-acetyl-mannosaminuronic acid. Arrows indicate synthesis direction. Double arrow indicates reaction reversibility 
of UDP-D-FucNAc to the membrane-anchored lipid carrier undecaprenyl-phosphate $\left(\mathrm{C}_{55} \mathrm{P}\right)$, yielding lipid $\mathrm{I}_{\text {cap }}$.

The second cytoplasmic reaction cascade generating the soluble precursor UDP-L-FucNAc involves the enzymes CapE, CapF and CapG, the enzymatic functions of which have already been elucidated biochemically ${ }^{13}$. The transferase CapL is assumed to further attach L-FucNAc to lipid $\mathrm{I}_{\text {cap }}$ leading to the formation of the second $\mathrm{CP}$ lipid intermediate, lipid $\mathrm{II}_{\text {cap }}$.

The third nucleotide-activated monosaccharide required for CP5 production, UDP-D-ManNAcA, is generated by the epimerase CapP and the dehydrogenase $\mathrm{CapO}^{7,14}$. The transmembrane protein CapI has been proposed to transfer the ManNAcA moiety to lipid $\mathrm{II}_{\text {cap }}$, thereby generating the final capsule precursor lipid III $_{\text {cap }}$. The $\mathrm{C}_{55} \mathrm{P}$-coupled trisaccharide is most likely further modified by the putative acetyltransferase $\mathrm{CapH}^{15}$, which catalyzes the $\mathrm{O}$-acetylation of L-FucNAc residues in position $\mathrm{C} 3$ in CP5 strains ${ }^{11}$. The complete, modified precursor is then translocated to the outer surface of the cell membrane, where polymerization is assumed to take place. These processes are proposed to be facilitated by the putative flippase CapK and the polymerase CapJ, respectively ${ }^{12,18}$. The attachment of $\mathrm{CP}$ precursors to the MurNAc ( $\mathrm{N}$-acetylmuramic acid) moiety of peptidoglycan is achieved by a yet unknown mechanism possibly involving a member of the LCP (LytR-CpsA-Psr) family of proteins ${ }^{20,21}$. This process likely releases the lipid carrier $\mathrm{C}_{55} \mathrm{P}$, which enters new synthesis cycles.

The fact that the undecaprenyl-phosphate carrier is found in limited amounts within the cell ${ }^{22}$ and required for the biosyntheses of diverse cell envelope components, like CP, WTA and $\mathrm{PG}^{2,3,18}$, makes a well-orchestrated spatial and temporal regulation of these processes crucial for the viability of the cell. The consequences arising from perturbation of this balanced biosynthetic network have been well exemplified for WTA biosynthesis. Interference with late WTA biosynthesis steps has been shown to be lethal, although the polymer per se is not crucial for viability ${ }^{23}$. Similarly, late stage genes are conditionally essential since they are dispensable for viability in an early gene ( $\operatorname{tar} O$ or $\operatorname{tar} A)$ deletion background, a phenomenon referred to as the "essential gene paradox" 24 . Inhibition of late WTA biosynthesis steps causes the accumulation of dead-end lipid-linked intermediates and thus depletes the cellular pool of $\mathrm{C}_{55} \mathrm{P}$ to critical levels impeding peptidoglycan biosynthesis, resulting in cell death ${ }^{25}$.

In $S$. aureus, the regulation of $\mathrm{CP}$ biosynthesis is not only achieved by differential gene expression ${ }^{26-28}$, but has additionally been linked to tyrosine phosphorylation ${ }^{16,17}$. Bacterial tyrosine kinases (BY-kinases) are widespread in bacteria and have multifaceted roles in bacterial exopolysaccharide production ${ }^{29}$. BY-kinases belong to the family of P-loop containing kinases ${ }^{30}$, whereby "P-loop" designates a characteristic amino acid sequence resembling the Walker A nucleotide binding motif. BY-kinases of Firmicutes are composed of two interacting polypeptides, a transmembrane activator protein and a cytoplasmic BYkinase $^{31}$. The cytoplasmic kinase carries a C-terminal tyrosine cluster that undergoes autophosphorylation in the presence of ATP. Based on studies in S. aureus and Streptococcus pneumoniae, the cytoplasmic kinase protein alone is not sufficient for phosphotransfer, but has to interact with the C-terminus of the transmembrane adaptor to undergo autophosphorylation $16,32,33$. Concomitantly, phosphate groups can be transferred to tyrosine residues of target proteins, thus modulating their activity. Two triplets of adjacent genes encoding for a transmembrane adaptor, a cytoplasmic BY-kinase, and a cognate phosphotyrosine phosphatase were identified in the genome of $S$. aureus serotype 5: The capA1/capB1/capC1 triplet (also referred to cap5A/cap5B/cap5C) is located at the 5 -end of the cap5 operon (Fig. 1a), whereas the highly similar capA2/capB2/capC2 triplet is found elsewhere on the bacterial chromosome $e^{12,16}$. So far, the cellular roles of the distinct CapAB complexes are not fully understood. Particularly, the nature of the stimulus that triggers CapAB signaling and the exact mode of signal transduction are still elusive.

In this study, we functionally reconstitute the entire $\mathrm{CP}$ biosynthetic reaction cascade generating the three membraneanchored CP precursors lipid $\mathrm{I}_{\text {cap }}$, lipid $\mathrm{II}_{\mathrm{cap}}$ and lipid $\mathrm{III}_{\mathrm{cap}}$, allowing us to identify crucial enzymatic check points, which are regulated by the tyrosine kinase CapA1B1 complex to control the consumption of essential precursors. Reconstitution of the membrane anchored CapA1 adaptor protein further reveals a yet elusive function. We show that CapA1 is a dual-function kinase activator/phosphodiesterase protein crucial for signaling and processing of the CP polymer. CapA1 interacts with lipid-bound $\mathrm{CP}$ precursors to catalyze the cleavage of the pyrophosphate linkage, releasing the essential lipid carrier $\mathrm{C}_{55} \mathrm{P}$. Moreover, we elucidate the principles of $\mathrm{CP}$ attachment to murein precursors in Gram-positive bacteria. We show that the transfer of the capsular phosphosugar moiety is conducted by a member of the LCP protein family and identify the acceptor substrate. We provide biochemical evidence that the attachment of the anionic precursor likely occurs on the level of the lipid-linked peptidoglycan precursor lipid II, and is facilitated in the presence of CapA1, indicating its cooperative functioning with LCP proteins.

\section{Results}

In vitro reconstitution of capsule biosynthesis. Homology searches with CapD and CapN suggested that both proteins are involved in the synthesis of the first soluble capsule precursor UDP-D-FucNAc ${ }^{18}$. More recently, CapD was characterized at the molecular level, and the enzymatic product was shown to be UDP-2-acetamido-2,6-dideoxy-D- $x y l o-4$-hexulose ${ }^{19}$. CapN is proposed to further convert the CapD reaction product to UDP-D-FucNAc by stereospecific reduction of the C-4 keto group $^{18}$.

As confirmed by capillary electrophoresis (CE) and mass spectrometry (MS), purified CapN (Supplementary Figure 1) catalyzed the NADPH-dependent conversion of the intermediate UDP-2-acetamido-2,6-dideoxy-D-xylo-4-hexulose to a sugar nucleotide species having a molecular mass of $\mathrm{m} / \mathrm{z} 590.4$ for the negatively charged molecule, consistent with the formation of UDP-D-FucNAc (Supplementary Figure 2). The subsequent transfer of the phosphosugar moiety of UDP-D-FucNAc to the lipid anchor $\mathrm{C}_{55} \mathrm{P}$ is thought to be catalyzed by the polyprenylphosphoglycosyltransferase CapM, to initiate the assembly of the lipid-anchored trisaccharide repeating units ${ }^{18}$. Incubation of purified CapM protein with $\mathrm{C}_{55} \mathrm{P}$ and UDP-D- $\left[{ }^{14} \mathrm{C}\right]$ FucNAc revealed the formation of a new radiolabeled lipid species, not present in the negative control (Fig. 2a). In comparison to $\mathrm{C}_{55} \mathrm{P}$ (Fig. 2a, lane $1 ; \mathrm{Rf}=0.95$ ), migration of the lipid product was retarded (lane $3 ; \mathrm{Rf}=0.87$ ), consistent with the addition of a sugar moiety. The product displayed the same $R_{f}$ value as determined for the WTA lipid intermediate undecaprenylpyrophosphoryl-D-GlcNAc (lipid III), which is structurally very similar to the proposed first CP lipid intermediate ${ }^{34}$. The newly formed purified lipid intermediate had a mass of $\mathrm{m} / z 1112.7058$ for the negatively charged molecule (Fig. 2b), which is consistent with the calculated neutral mass for undecaprenyl-pyrophosphoryl-D-FucNAc (lipid I $\mathrm{I}_{\text {cap }}$ ) of 1113.7162. Moreover, the GT-B type $^{35}$ glycosyltransferase CapL was able to use lipid $\mathrm{I}_{\text {cap }}$ as acceptor substrate and to catalyze the addition of the second ${ }^{14} \mathrm{C}$ labeled sugar moiety L-FucNAc, yielding lipid $\mathrm{II}_{\text {cap }}$ (Fig. 2a, lane $4 ; \mathrm{Rf}=0.83)$. Mass spectrometry analysis of lipid $\mathrm{II}_{\text {cap }}$ revealed a mass of $\mathrm{m} / z 1299.7896[\mathrm{M}-\mathrm{H}]^{-}$matching the 
a

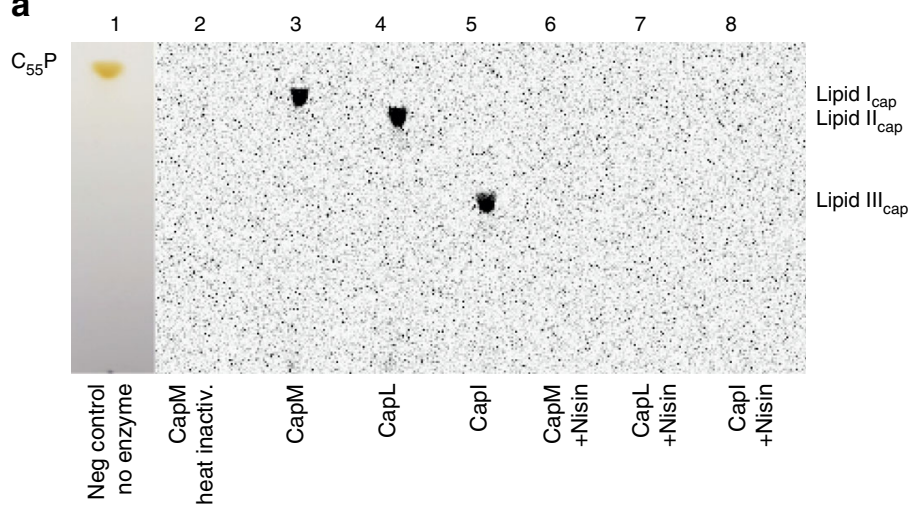

C

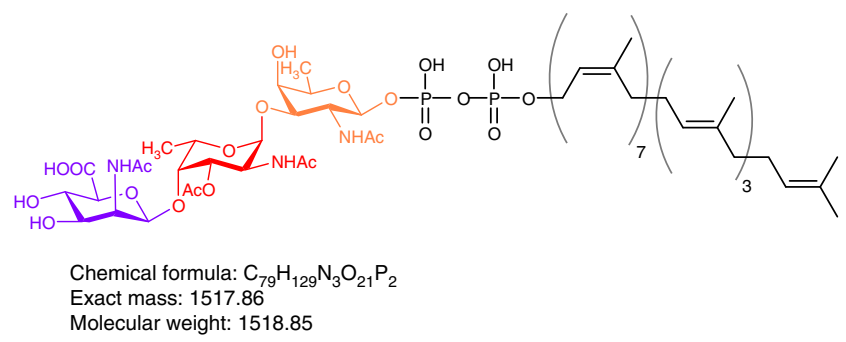

b

\begin{tabular}{llccc}
\hline Formula & Mass calc. & $\begin{array}{c}\text { Mass calc. } \\
{[\mathrm{M}-\mathrm{H}]^{-}}\end{array}$ & $\begin{array}{c}\text { Mass measured } \\
{[\mathrm{M}-\mathrm{H}]^{-}}\end{array}$ \\
\hline Lipid I $\mathrm{I}_{\text {cap }}$ & $\mathrm{C}_{63} \mathrm{H}_{105} \mathrm{O}_{11} \mathrm{P}_{2} \mathrm{~N}$ & 1113.7162 & 1112.7079 & 1112.7058 \\
Lipid II & $\mathrm{C}_{71} \mathrm{H}_{118} \mathrm{O}_{15} \mathrm{P}_{2} \mathrm{~N}_{2}$ & 1300.8007 & 1299.7924 & 1299.7896 \\
\hline
\end{tabular}
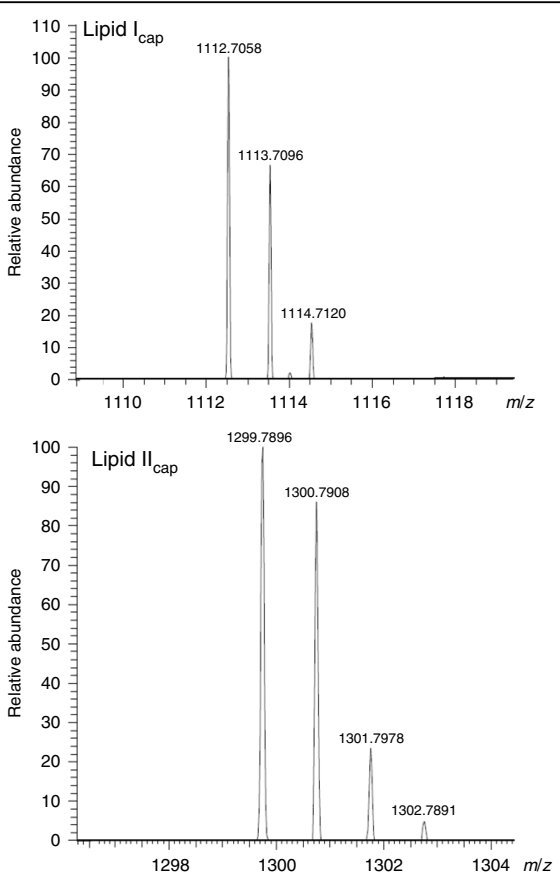

Fig. 2 Synthesis of CP lipid intermediates. Glycosyltransferases (GT) CapM, CapL and Capl catalyze the in vitro synthesis of CP lipid intermediate lipid I $\mathrm{Iap}$ $\left(C_{55}\right.$ PP-D-FucNAc), lipid II $I_{\text {cap }}\left(C_{55}\right.$ PP-D-FucNAc-L-FucNAc) and lipid III $I_{\text {cap }}\left(C_{55}\right.$ PP-D-FucNAc-L-FucNAc-D-ManNAcA), respectively. a Purified GTs were incubated with the respective purified acceptor lipid substrate in the presence of radiolabeled UDP-activated sugars. After extraction with BuOH/PyrAc, synthesis products were analyzed by TLC and phosphoimaging. Counterstain with iodide (lane 1) was used to visualize the migration behavior relative to $\mathrm{C}_{55} \mathrm{P}$. Nisin, known to bind to the pyrophosphate-sugar moiety of cell wall lipid intermediates was added posterior and formed complexes with all lipid ${ }_{\text {cap }}$ intermediates that are not extracted from the reaction mixture. Heat-inactivated CapM was used as a negative control. $\mathbf{b}$ ESI-MS-analysis of lipid $\mathrm{I}_{\text {cap }}$ and lipid $I_{\text {cap. }}$ The peaks at $\mathrm{m} / \mathrm{z} 1112.70$ and 1299.78 correspond to the negatively charged lipid $\mathrm{I}_{\text {cap }}$ and lipid II cap molecules, respectively. c Structure of the

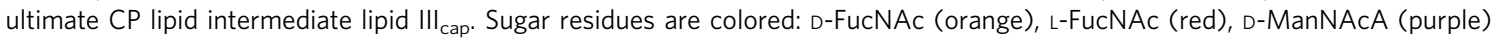

calculated neutral mass of $\mathrm{m} / z 1300.801$ for undecaprenylpyrophosphoryl-D-FucNAc-L-FucNAc.

Conversion to the ultimate lipid-linked CP precursor lipid III $_{\text {cap }}$ was achieved by the attachment of a ${ }^{14} \mathrm{C}$-D-ManNAcA residue to lipid $\mathrm{II}_{\text {cap }}$, catalyzed by the glycosyltransferase CapI (Fig. 2a, lane $5 ; \mathrm{Rf}=0.53$ ). Mass spectrometric analysis of lipid III $_{\text {cap }}$ did not yield any signal, presumably due to reduced ionization with addition of further sugar moieties. However, in line with the altered migration behavior of the different $\mathrm{CP}$ intermediates on TLC (Fig. 2a, lanes 3-5) resulting from the consecutive sugar residue addition, the lantibiotic nisin formed extraction-stable complexes with all $\mathrm{C}_{55} \mathrm{P}$-containing $\mathrm{CP}$ precursors further validating their identity (Fig. 2a, lanes 6-8). Taken together, these findings confirm the proposed functions of the different glycosyltransferases (GT) and demonstrate that CapM functions as priming GT, initiating the synthesis of membranebound $\mathrm{CP}$ precursors by coupling the first sugar moiety to the lipid carrier $\mathrm{C}_{55} \mathrm{P}$.

CapM is homologous to a wide range of GTs from various pathogens (Supplementary Figure 3a), which catalyze the transfer of UDP-activated sugars to $\mathrm{C}_{55} \mathrm{P}^{36,37}$. Prediction of transmembrane topology (http://www.cbs.dtu.dk/services/TMHMM/) and comparison with hydrophobicity plots (ProtScale ${ }^{38}$ ) of homologous GTs predicts that CapM is anchored to the cytoplasmic membrane by a 28 -amino acid long $\alpha$-helical transmembrane domain that is linked to a large C-terminal catalytic domain located in the cytoplasm (Supplementary Figure 3b). In contrast to the in silico predicted single-pass transmembrane (TM) helix geometry, structural analysis of the homologous PglC of Campylobacter concisus suggests that the TM segment adopts an unusual architecture ${ }^{37}$. Structural modelling of CapM revealed a similar architecture (Supplementary Figure 3c), in which the TM segment is broken into two helices ( $A$ and $B$ ) with an interhelix angle of $118^{\circ}$ by a Ser-Pro motif (Supplementary Figure 3c), suggesting a similar membrane embedment as PglC. Shared structural features further include a conserved Arg residue (Arg3) positioned at the membrane interface as well as a catalytic Asp-Glu dyad and a strictly conserved PRP motif (residues 110-112 in CapM) in the cytoplasmic GT domain, involved in $\mathrm{Mg}^{2+}$ and substrate binding, respectively ${ }^{37}$ (Supplementary Figure 3). CapM activity in vitro depends on the presence of $\mathrm{MgCl}_{2}$, and was completely lost in the presence of EDTA (Supplementary Figure 4a), as reported for homologous glycosyltransferases ${ }^{36,37,39}$.

Interestingly, CapM was inhibited in the presence of tunicamycin (Supplementary Figure 4a), a nucleoside antibiotic shown to inhibit members of the UDP-HexNAc-1-phosphate transferase family (i.e., WecA, MraY or TarO) through competitive binding to the DDxxD $\mathrm{Mg}^{2+}$-binding site ${ }^{39}$. Tunicamycin had an $\mathrm{IC}_{50}$ of $129 \mu \mathrm{M}$ for CapM, which is in the range determined for PglC of $C$. jejuni $\left(\mathrm{IC}_{50}=\text { of } 100 \mu \mathrm{M}\right)^{40}$. The lower potency of tunicamycin for these monotopic GTs compared to the polytopic WecA (IC ${ }_{50}$ of $\left.11 \mathrm{nM}\right), \mathrm{MraY}\left(\mathrm{IC}_{50}\right.$ of $\left.\sim 22.5 \mu \mathrm{M}\right)$ or TarO ( $\mathrm{IC}_{50}$ of $\left.\sim 59 \mathrm{nM}\right)$ may likely result from the distinct 
architectures and catalytic sites. Since we observed a major impact of anionic phospholipids (dioleoylphosphatidylglycerol and cardiolipin) on the enzymatic activity of CapM, we further investigated the effect of different detergents. However, CapM showed lower activity in the presence of detergents or DMSO indicating that the enzyme preferred the native phospholipid environment (Supplementary Figure $4 \mathrm{~b}$ ).

CapAB phosphorylation positively modulates CapM and CapE activity. Full-length integral membrane proteins CapA1 and CapA2 and cytoplasmic kinases CapB1 and CapB2 were overexpressed as $\mathrm{His}_{6}$-tag fusion proteins, purified by Ni-NTA chromatography (Supplementary Figure 1), and utilized to reconstitute $\mathrm{CapAB}$ tyrosine kinase activity in vitro in the presence of $\gamma$-labeled $\left[{ }^{33} \mathrm{P}\right] \mathrm{ATP}$. CapB2 autophosphorylation was effectively activated by full-length CapA1 or CapA2 (Fig. 3a) producing a single radiolabeled band with an apparent molecular mass of $25.3 \mathrm{kDa}$, corresponding to phosphorylated CapB2 (lanes 3 \& 4). In contrast, the negative control in which a CapA activator was absent (Fig. 3a, lane 1 and 2, Supplementary Figure 5a) showed no autophosphorylation. In line with previous findings ${ }^{16}$, phosphorylation of the individual kinase protein CapB1 was neither observed with CapA1 nor with CapA2 (Fig. 3a, lanes $5 \&$ 6, Supplementary Figure 5a, lanes $6 \& 7$ ). However, when CapB1 was fused to its cognate activator CapA1, the recombinant chimera CapAB full-length fusion protein $(50.2 \mathrm{kDa})$ efficiently autophosphorylated in the presence of $\gamma$-labeled $\left[{ }^{33} \mathrm{P}\right] \mathrm{ATP}$, demonstrating functionality of CapB1 a

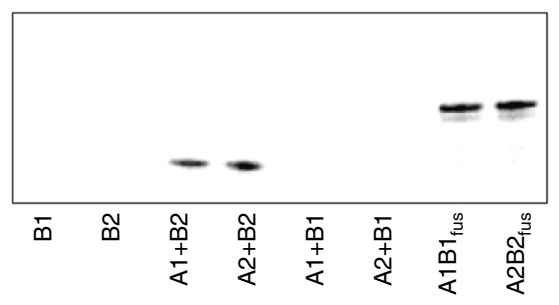

b

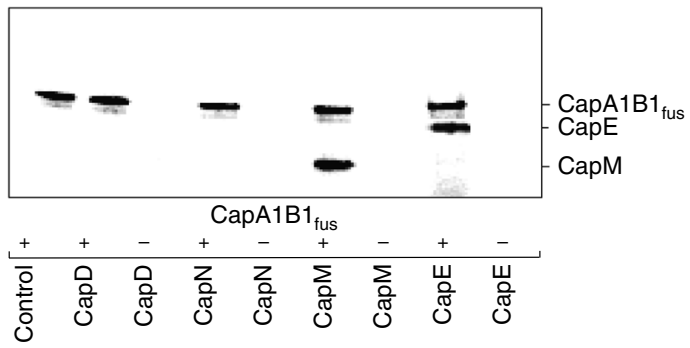

C
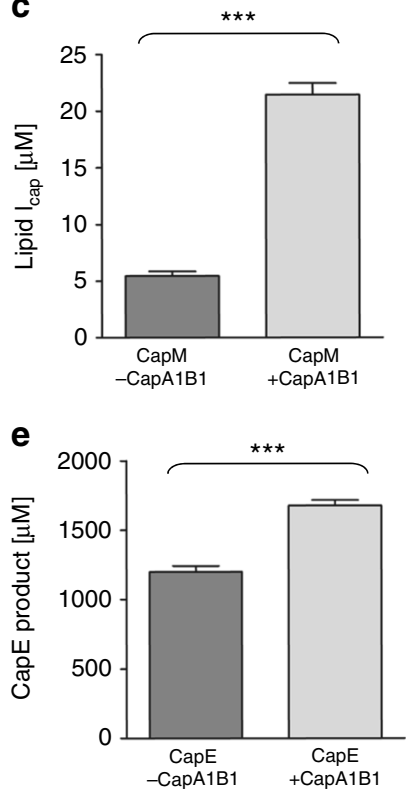

d

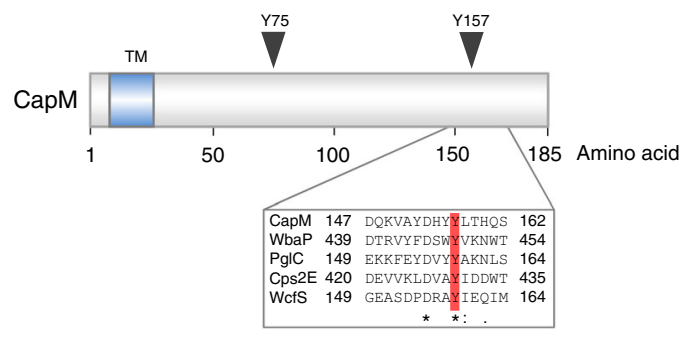

$\mathbf{f}$

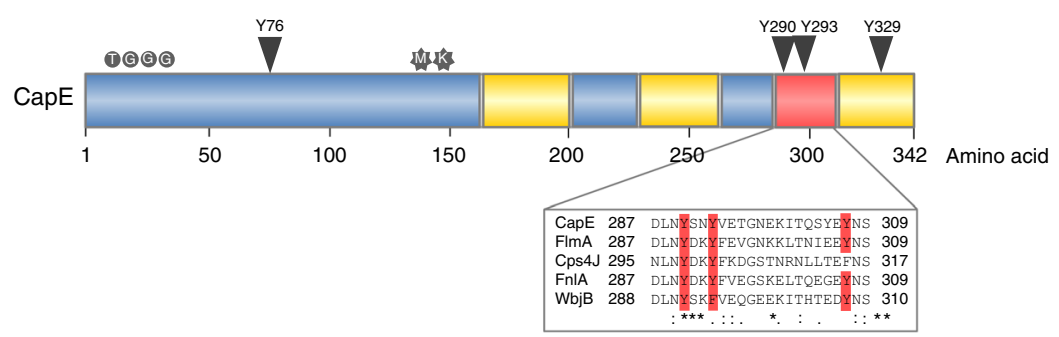

Fig. 3 Tyrosine phosphorylation positively controls CapM and CapE activity. a CapAB autokinase activity was assayed in the presence of [33P]ATP using either native proteins or kinase activator $\mathrm{CapA} 1$ and tyrosine kinase $\mathrm{CapB1}$ fused into a single polypeptide (CapA1B1 fus $)$. Phosphotransfer was analyzed by SDS-PAGE and phosphoimaging. b CapM and CapE are phosphorylated by the CapA1B1 fus tyrosine kinase complex. Putative target proteins ( $3 \mu \mathrm{g}$ ) were assayed for phosphotransfer in the presence and absence of CapA1B1 fus. CapA1B1 fus, 50.2 kDa; CapB2, 25.3 kDa; CapE, 38.6 kDa; CapD, 69.1 kDa; CapM, $21 \mathrm{kDa}$; CapN, $33.7 \mathrm{kDa}$. c Impact of CapA1B1 kinase activity on CapM glycosyltransferase activity. CapM was incubated in presence of either active CapA1B1 (light grey) or heat-inactivated CapA1B1 (dark grey) with UDP-D- $\left[{ }^{14} \mathrm{C}\right] \mathrm{FucNAc}$ and $\mathrm{C}_{55} \mathrm{P}$. After extraction with BuOH/PyrAc, reactions were analyzed by TLC and phosphoimaging. d Topology and CapAB phosphorylation site (arrow) of CapM. The protein is anchored in the membrane by one reentrant transmembrane domain. The catalytic domain is located in the cytoplasm. Sequence alignment of homologous proteins containing the conserved Tyr phosphorylation site (red) is boxed. (from top to bottom: S. aureus, Salmonella enterica, Campylobacter concisus, Streptococcus pneumoniae and Bacteroides fragilis). e Impact of CapA1B1 kinase activity on CapE-mediated substrate conversion. CapE was incubated in presence of either active CapA1B1 (light grey) or heat-inactivated CapA1B1 (dark grey) with UDP-D-GIcNAc. Reactions were analyzed by CE. f Mapping of CapE phosphorylation sites. Tyr phosphorylation sites are marked by arrows. Cofactor binding site, substrate-binding site and the mobile loop are marked blue, yellow and red, respectively. The active site motif MxxxK (stars) and the cofactor binding motif TGxxGxxG (circles) are highlighted. A sequence alignment of the mobile latch (red) of selected homologous proteins from different species is boxed (from top to bottom: S. aureus, Bacillus cereus, Streptococcus pneumoniae, Enterococcus faecium, Pseudomonas aeruginosa). Experiments were performed in triplicate. The error bars represent the \pm standard deviation (SD) from three biological replicates. Statistical significance was analyzed by an unpaired $t$ test $\left.{ }^{\star \star \star} p<0.005\right)$ 
(Fig. 3a, lane 7). A full-length CapA2B2 fusion protein, which was constructed for reasons of comparison, showed comparable autokinase activity (lane 8 ). This finding contradicts recent suggestions that CapB1 might be a pseudokinase devoid of catalytic activity $^{41}$.

CapA1B1-mediated phosphotransfer on putative target proteins involved in CP biosynthesis revealed tyrosine phosphorylation of the glycosyltransferase CapM $(21 \mathrm{kDa})$ and the dehydratase CapE (38.6 kDa) (Fig. 3b, Supplementary Figure 5b). In contrast, recombinant CapD, CapN, CapF, CapG or CapL were not phosphorylated (Fig. 3b, Supplementary Figure 5c). The opposing PHP class phosphatases CapC1 and CapC2 were able to antagonistically dephosphorylate CapB kinase and target proteins (Supplementary Figure 5d).

Phosphorylation of CapM by the CapA1B1 fusion increased lipid $I_{\text {cap }}$ synthesis 4-fold (Fig. 3c), showing that CapAB-mediated signaling stimulates the priming step of CP biosynthesis in $S$. aureus by enhancing the rate of CapM-catalyzed glycosyl transfer and thus likely controlling the consumption of the shared carrier $\mathrm{C}_{55} \mathrm{P}$.

In silico phosphorylation site prediction (NetPhos 3.1$)^{42}$ identified tyrosines 75 and 157 as putative phosphosites in CapM (Fig. 3d). The highly conserved Tyr157 appears to be the primary phosphorylation site in CapM, since tyrosine phosphorylation and the concomitant stimulatory effect on the catalytic activity was completely abolished in the CapM_Y157F mutant (Supplementary Figure 6a,b). In contrast, changing Tyr75 to Phe had only a minor effect on the protein's ability to be catalytically activated by CapA1B1-mediated phosphotransfer. Both mutant proteins retained catalytic activity comparable to wild-type CapM in the absence of CapA1B1 (Supplementary Figure 6b) showing that Tyr157 represents the crucial regulatory CapAB phosphorylation site on CapM.

The CapA1B1-mediated stimulation of CapM activity is also reflected by enzyme kinetics, revealing a $\mathrm{Km}$ value of $2,309 \pm 311.8 \mu \mathrm{M}$ for UDP-D-FucNAc and a $V_{\max }$ value of $0.5559 \pm 0.02645 \mathrm{pmol} \mathrm{min}^{-1} \mu \mathrm{g}^{-1}$ in the absence of CapA1B1 and a lowered $K_{\mathrm{m}}$ value of $996.2 \pm 136.2 \mu \mathrm{M}$ and an increased $V_{\max }$ of $1.0688 \pm 0.0358 \mathrm{pmol} \mathrm{min}^{-1} \mu \mathrm{g}^{-1}$ in the presence of CapA1B1 (Supplementary Figure 6c). Moreover, a CapM phosphomimetic in which Tyr157 was exchanged to Glu displayed a $K_{\mathrm{m}}$ value of $1383 \pm 267.3 \mu \mathrm{M}$ and a $V_{\max }$ value of $0.8625 \pm 0.04708 \mathrm{pmol} \mathrm{min}^{-1} \mu \mathrm{g}^{-1}$ in the absence of CapA1B1, substantiating the stimulatory impact of CapM phosphorylation on Tyr157 (Supplementary Figure 6c).

In vitro kinase assays further identified CapE as a CapA1B1 target (Fig. 3e). Tryptic fragments of in vitro phosphorylated CapE were analyzed by nanoscale liquid chromatography coupled to tandem mass spectrometry (nanoLC-MS/MS), and Tyr76 of the CapE protein was found to be phosphorylated (Supplementary Figure 7a). This residue is located in proximity to the conserved TGxxGxxG motif required for cofactor binding and to the MxxxK catalytic site ${ }^{43}$ (Fig. 3f). Three other phosphotyrosine residues (Tyr290, Tyr293 and Tyr329), were mapped to a C-terminal loop of CapE (Fig. 3f; Supplementary Figure 7a). In CapE in vitro assays with active CapAB, the conversion of UDP-D-GlcNAc was increased by $40 \%$, demonstrating that the CapE catalyzed reaction is positively modulated through phosphorylation (Fig. 3e).

The role of the potential regulatory CapAB phosphorylation sites was probed in a site-directed mutagenesis study. As determined by CE quantification, replacing either Tyr76 of CapE or the three C-terminal tyrosine residues Tyr290, Tyr293, and Tyr329 with Phe diminished the activating effect of CapA1B1 on CapE. The stimulatory effect of CapA1B1 on the conversion of UDP-GlcNAc was completely abolished when all four tyrosine residues were mutated to Phe, confirming their role as regulatory phosphorylation sites on CapE (Supplementary Figure 7b).

CapA1 is a dual-function phosphodiesterase/kinase activator protein. When purified lipid $\mathrm{I}_{\text {cap }}$ was incubated in the presence of CapA1, an additional lipid band was detected by TLC analysis (Fig. 4a), which was not visible in the negative control with heatinactivated CapA1, indicating enzymatic conversion of the first $\mathrm{CP}$ lipid precursor. Intriguingly, the reaction product displayed an identical $\mathrm{R}_{\mathrm{f}}$ value as the lipid carrier $\mathrm{C}_{55} \mathrm{P}$. MALDI-TOF MS analysis determined a mass of $\mathrm{m} / z 845.610$ (negative mode; $[\mathrm{M}-\mathrm{H}]^{-}$) for the purified lipid product, confirming that CapA1 is able to catalyze the cleavage of the pyrophosphate linkage within the lipid $\mathrm{I}_{\text {cap }}$ intermediate to release $\mathrm{C}_{55} \mathrm{P}$ (Fig. $4 \mathrm{~b}$ ). Of note, CapA1 was unable to cleave the peptidoglycan precursor lipid $\mathrm{II}_{\mathrm{PG}}$, but was able to hydrolyze the WTA precursor lipid III (Supplementary Figure 8). The facts that CapA1 interacts with lipid-linked CP precursors, and exhibits phosphodiesterase activity towards lipid $\mathrm{I}_{\text {cap }}$, implies additional functions for this protein in CP biosynthesis, polymerization and attachment processes that go beyond being a mere "transmembrane activator". Importantly, no cleavage of lipid $\mathrm{I}_{\text {cap }}$ or lipid III $_{\text {WTA }}$ was observed with the CapA1 paralogue CapA2 (Fig. 4a), revealing functional differences between the two proteins.

CapA1 is anchored to the cytoplasmic membrane via two transmembrane domains flanking an outside loop comprising 130 amino acids (Fig. 4c), which likely represents a dual function sensory/catalytic domain involved in recognition and processing of membrane-bound CP precursors.

As evidenced in previous research, CapA1 is crucial for efficient capsule formation in $S$. aureus ${ }^{44}$. Corroborating these studies, complementation with pCapA1 in trans enhanced CP production in the serotype 8 strain MW2 (Fig. 4d). Strain MW2 carries a frameshift mutation in capA1 (cap5A) that results in expression of a truncated version (171 aa) of the full-length gene product $(222 \mathrm{aa})^{45}$. In contrast, deletion of capA2 in $S$. aureus Newman did not affect in vivo CP production (Fig. 4d). Similarly, the deletion of cognate $\mathrm{CapB}$ kinases revealed, that $c a p B 1$ but not $c a p B 2$ is required for $\mathrm{CP}$ production (Supplementary Figure 7c). In spite of the functional redundancy and overlapping protein target specificities of CapA1B1 and CapA2B2 observed in our in vitro phosphorylation assays, the two kinase complexes clearly have distinct roles in cell physiology.

In contrast to CapA1, CapA2 is not encoded within the cap5 operon, and does not exhibit phosphodiesterase activity towards lipid $\mathrm{I}_{\text {cap }}$, which led us to conclude that CapA2 has a distinct function in the biosynthesis of the bacterial cell envelope. More recently, the $S$. aureus exopolysaccharide poly- $N$-acetyl- $\beta-(1,6)$ glucosamine (PNAG; also referred to as polysaccharide intercellular adhesin) has been reported to bind to the CapA2 receptor loop ${ }^{46}$, suggesting that PNAG might represent a molecular signal detected by CapA2. We therefore investigated the influence of purified PNAG and CP5 on the autophosphorylation rate of the CapAB complexes. As revealed by in vitro kinase assays, CapA2-induced autophosphorylation of CapB2 was inhibited in the presence of PNAG, as well as in the presence of CP5, in a concentration-dependent manner (Supplementary Figure 9). In contrast, CapA1-induced phosphorylation of CapB2 was not diminished, indicating that the observed modulatory effect of exopolysaccharide molecules is mediated via an interaction with the transmembrane activator protein CapA2, and not via a direct inhibitory interaction with the protein kinase CapB2. This finding further substantiates differential roles for CapA1 and CapA2 in cell envelope biosynthesis. 
a

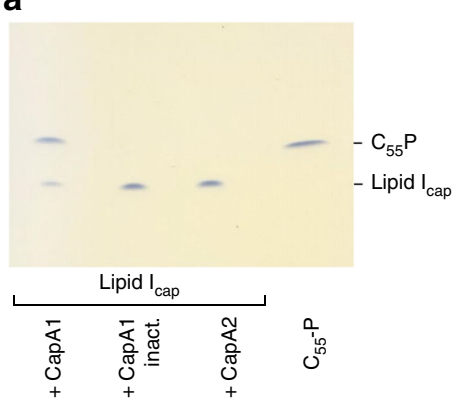

C
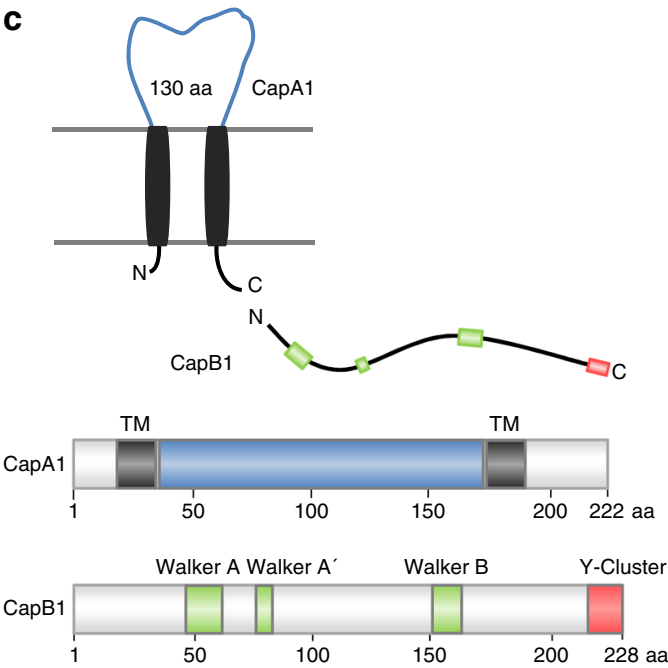

b

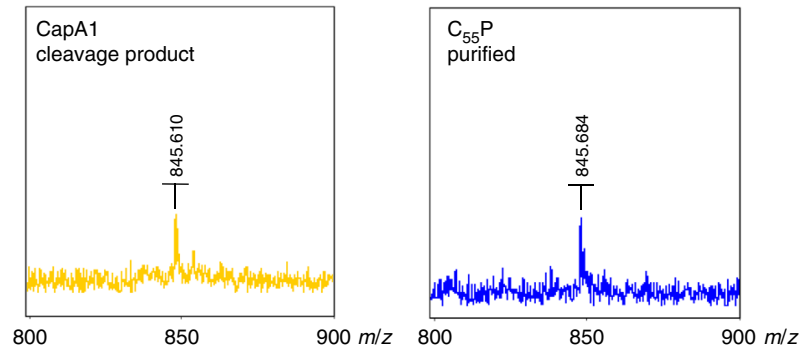

d
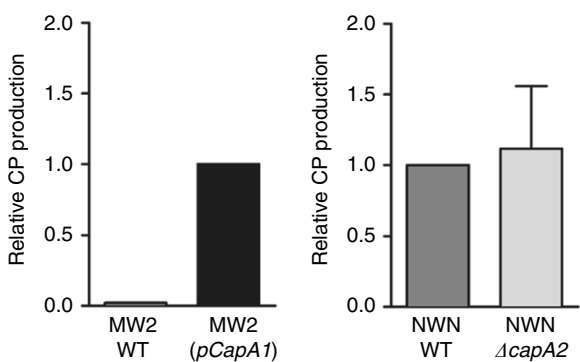

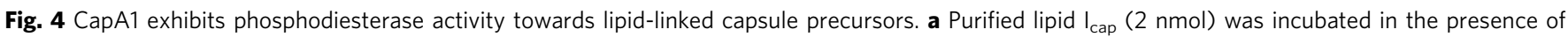

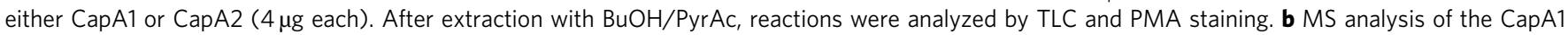
cleavage product isolated via preparative TLC. MALDI-TOF MS spectra were obtained with a Biflex III instrument running in negative mode. The $m / z$ of 845.61 corresponds to the singly charged $C_{55} P$ molecule, which has a measured mass of 845.68 and a calculated neutral mass of 846.67 . $\mathbf{c}$ CapA1 is

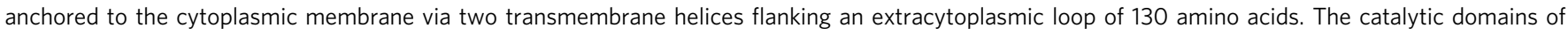

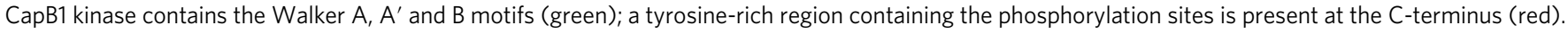
d ELISA-based quantification of CP production in S. aureus MW2 complemented in trans with pCapA1 (left) and S. aureus Newman wild type and a $\triangle$ capA2 deletion mutant (right). Experiments were performed in triplicate. The error bars represent the mean \pm SEM from three biological replicates

LcpC catalyzes the ligation of CP to lipid II. Structural analysis and phenotypic studies suggest that members of the LCP protein family catalyze the transfer of undecaprenyl-linked intermediates onto the C6-hydroxyl function of $\mathrm{N}$-acetylmuramic acid in PG, thereby promoting attachment of WTA and CP in Gram-positive bacteria $^{20,47}$. S. aureus encodes three LCP enzymes with semiredundant functions ${ }^{21}$. As deduced from knockout mutant studies, CP attachment is preferentially catalyzed by LcpC, though LcpA and LcpB may partially compensate for the loss of LcpC 21 .

To investigate the proposed role of $\mathrm{LcpC}$ in vitro purified $\left[{ }^{14} \mathrm{C}\right]$ lipid $\mathrm{I}_{\text {cap }}$ was incubated with purified LcpC protein and lipid $\mathrm{II}_{\mathrm{PG}}$ as a potential acceptor substrate. In this setup, LcpC was able to catalyze cleavage of the donor substrate lipid $\mathrm{I}_{\text {cap }}$ and catalyze attachment of the phosphoryl-sugar moiety to the ultimate PG precursor lipid II (Fig. 5a), resulting in a reaction product $\left(\mathrm{LII}_{\mathrm{PG}}-{ }^{14 \mathrm{C}} \mathrm{CP}\right)$ that migrates slower on TLC. Identical results were obtained when $\left[{ }^{14} \mathrm{C}\right]$-labeled lipid $\mathrm{II}_{\mathrm{PG}}$ and non-labeled lipid $\mathrm{I}_{\text {cap }}$ were used as the reaction substrates (Fig. 5b), further verifying the identity of the ligation product. Quantitative analysis revealed that $\sim 50 \%$ of the CP lipid precursor was attached to lipid II (Fig. 5c). Strikingly, the LcpC-mediated transferase reaction was significantly enhanced when CapA1 was included in the reaction mixture, indicating cooperative action of the transmembrane activator/phosphodiesterase protein and LcpC (Fig. 5c).
CapA1 alone was unable to catalyze the attachment of the $\mathrm{CP}$ precursor (Supplementary Figure 10a). Of note, LcpC was able to catalyze hydrolytic cleavage of lipid $\mathrm{I}_{\text {cap }}$, but not of lipid $\mathrm{II}_{\mathrm{PG}}$, a reaction that would likely be deleterious in vivo (Supplementary Figure 10b, c).

Interestingly, $\mathrm{PBP} 2$ added posterior to the $\mathrm{LcpC}$ reaction efficiently catalyzed the transglycosylation of lipid $\mathrm{II}_{\mathrm{PG}-\mathrm{CP}}$ (Fig. 5a, b). The resulting polymerized $\mathrm{PBP}$ reaction product is not extracted from the reaction mixture, thus lipid bands vanish from the TLC. The antibiotic moenomycin, known to inhibit the PBP2 catalyzed transglycosylation, fully blocked the polymerization reaction (Fig. 5b). Importantly, LcpC was further able to efficiently transfer the disaccharide of lipid $\mathrm{II}_{\text {cap }}$ and the trisaccharide of the ultimate CP lipid intermediate lipid III $_{\text {cap }}$ to lipid $\mathrm{II}_{\mathrm{PG}}$, as evidenced by the altered migration of the respective ligation products $(\mathrm{Rf}=0.26$ and $\mathrm{Rf}=0.22$, respectively). Efficient processing of ligation products by $\mathrm{PBP} 2$ shows that $\mathrm{CP}$ attachment could occur in parallel to PG assembly (Fig. 5d).

Interestingly, antisense-RNA mediated depletion of CapA1 in a triple $\Delta l c p$ mutant was lethal, indicating that CapA1 hydrolysis of $\mathrm{C}_{55} \mathrm{P}$-coupled CP and WTA precursors contributes to rescue $S$. aureus from the accumulation of toxic intermediates (Supplementary Figure 10d) and further supports a functional link between CapA1 and LcpC. 
a

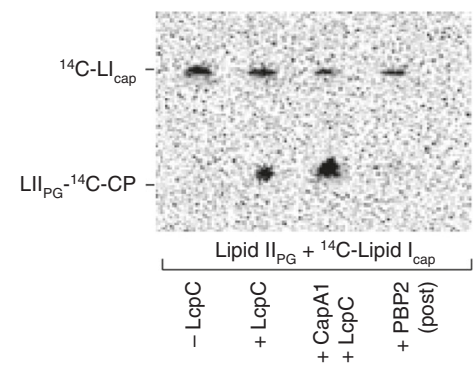

b

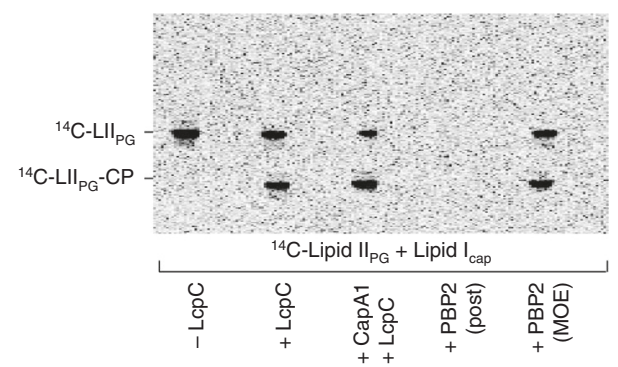

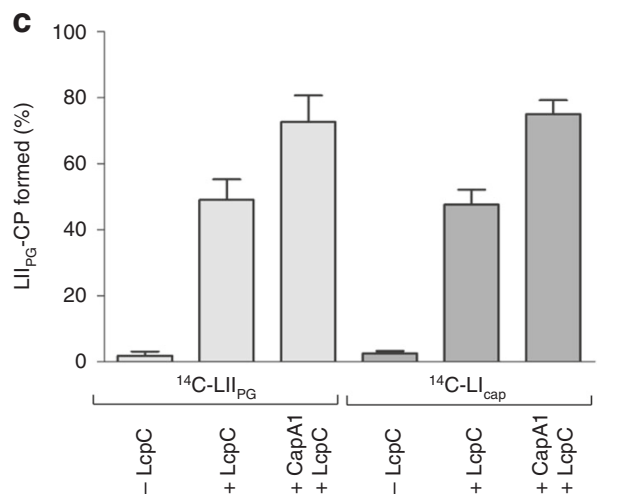

d

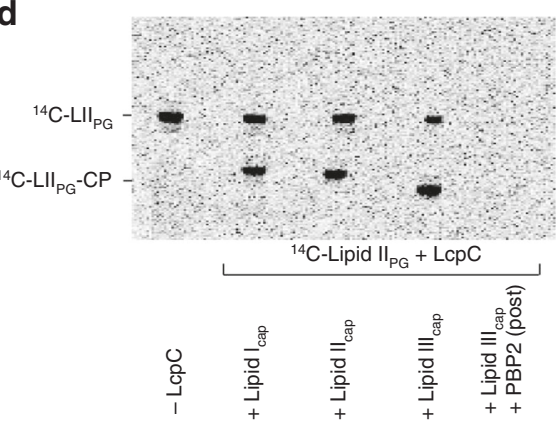

Fig. 5 Attachment of CP to the ultimate PG building block lipid $I_{P G}$ is mediated by $L c p C$. a $\left[{ }^{14} C\right]$ lipid $I_{\text {cap }}$ and lipid $I_{P G}$ or $\mathbf{b}$ lipid $I_{c a p}$ and $\left[{ }^{14} C\right]$ lipid $I_{P G}$ were incubated with $\mathrm{LCpC}$ in the absence or presence of CapA1. The resulting reaction product ( $\mathrm{L} \|_{\mathrm{PG}}-\mathrm{CP}$; bold) exhibits an altered migration behavior on the TLC compared to the individual substrates, lipid $\|_{\mathrm{PG}}$ and lipid $\mathrm{I}_{\text {cap. }}$. PBP2 catalyzes the transglycosylation of the $\mathrm{LcpC}$ reaction product $\mathrm{L} \mathrm{I}_{\mathrm{PG}}-\mathrm{CP}$ (lane 4 ) resulting in the polymerization of radiolabeled lipid $I_{P G}-\mathrm{CP}$ into hydrophilic glycan strands that retain in the water phase and are not extracted after BuOH/PyrAc treatment resulting in disappearance of the corresponding band on the chromatogram. The PBP2 catalyzed conversion is inhibited in the presence of the antibiotic moenomycin (MOE; $10 \mu \mathrm{M}$ ) (B, lane 5). c Quantification of lipid $I_{\text {cap }}$ attachment to lipid IIPG. Radiolabeled bands were quantified by phosphoimaging. Experiments were performed in triplicate. The error bars represent the \pm SD from three biological replicates. $\mathbf{d}$ LcpC catalyzes the attachment of lipid II cap (lane 3) and the ultimate CP lipid intermediate lipid III $I_{\text {cap }}$ (lane 4) and the final reaction product is processed by PBP2 added posterior to the $\mathrm{LcpC}$ reaction (lane 5). Representative TLC of 2 independent experiments

Capsule biosynthesis is negatively controlled by PknB. As evidenced in previous research, the eukaryotic serine/threonine kinase (ESTK) $\mathrm{PknB}$ of $S$. aureus is involved in the regulation of cell envelope biogenesis processes ${ }^{48-50}$ and was more recently shown to sense lipid $\mathrm{II}_{\mathrm{PG}}{ }^{51}$. Testing CapM in an in vitro kinase assay showed PknB-mediated phosphorylation of the priming GT (Fig. 6a). Moreover, quantitative analysis of the in vitro CapMcatalyzed reaction revealed an up to $30 \%$ decrease in lipid $\mathrm{I}_{\text {cap }}$ synthesis when purified PknB kinase and ATP were included in the reaction mixture, compared to a control reaction containing heat-inactivated $\mathrm{PknB}$, suggesting that ESTK signaling negatively controls the activity of CapM (Fig. 6b). LC/MS-analysis revealed PknB-mediated phosphotransfer onto Thr67, Thr128 and Thr134 of CapM (Supplementary Figure 11). Thr134 is highly conserved among homologous bacterial glycosyltransferases, located in proximity of the membrane interface and the amphipathic helices $\mathrm{D}$ and I (Supplementary Figure 3c) and may thus be the most promising candidate for a regulatory $\mathrm{PknB}$-phosphorylation site on CapM.

Intriguingly, the kinase protein CapB1 was also identified as potential phosphorylation target of PknB (Fig. 6c), indicating the possibility of kinase cross-talk. To further explore this finding, the autophosphorylation activity of the CapA1B1 complex was assessed in the presence of $\mathrm{PknB}$. The overall phosphorylation intensity of CapA1B1 was decreased by $34 \%$ in the presence of active PknB (Fig. 6d), compared to the heat-inactivated negative control, showing that PknB-mediated serine/threonine phosphorylation inhibits CapB1 autophosphorylation on tyrosine. Thr8 was identified as the specific phosphorylation site on CapB1 by
LC/MS analysis (Supplementary Figure 12a) and a CapA1B1 $1_{\text {fus }}$ phosphomimetic in which this Thr was exchanged to Glu resulted in a significantly reduced CapB1 autophosphorylation (Supplementary Figure $12 \mathrm{~b}, \mathrm{c}$ ), suggesting that PknB-mediated phosphorylation interferes with the CapB1 activation by CapA1 11,52 . In contrast, an exchange of Thr8 to Ala did not affect CapA1B1 $1_{\text {fus }}$ autophosphorylation (Supplementary Figure 12b,c).

To determine whether the observed inhibitory effect of PknB in vitro would translate into an in vivo effect, we compared CP5 production in $S$. aureus Newman and in an isogenic $p k n B$ deletion mutant. Consistent with the proposed role of $\mathrm{PknB}$ as negative regulator of $\mathrm{CP}$ biosynthesis, the amount of cellassociated CP5 was 5-fold higher in the $p k n B$ mutant compared to the parental strain and complementation of the $\Delta p k n B$ mutant in trans restored CP production to wild-type level (Fig. 6e).

The in vitro and in vivo data reveal that $\mathrm{PknB}$ signaling serves to reduce CapM GT activity, as well as CapA1B1 autokinase activity, allowing for a shutdown of CP production likely ensuring a sufficient supply of precursors for PG formation, and thus maintenance of cell wall architecture and function.

\section{Discussion}

Biosynthesis of the $S$. aureus CP shows similarity to the syntheses of PG and WTA, in that all pathways share a pool of essential precursors, i.e. the lipid carrier undecaprenyl-phosphate and UDP-D-GlcNAc ${ }^{1,53}$. Since the availability of $\mathrm{C}_{55} \mathrm{P}$ within the bacterial cell is limited ${ }^{22}$, distribution and prioritization for the alternate metabolic pathways need to be tightly controlled in time and space to ensure bacterial viability. 
a

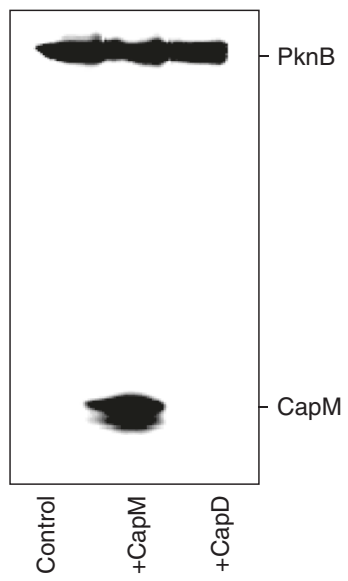

b

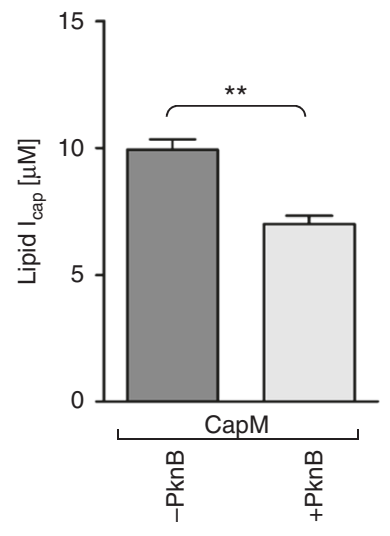

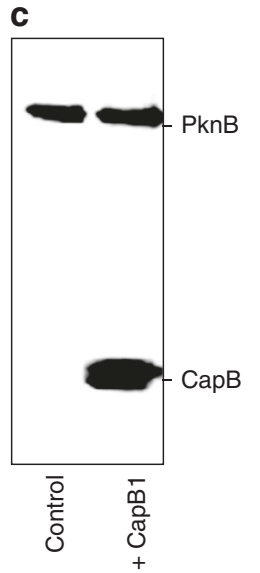
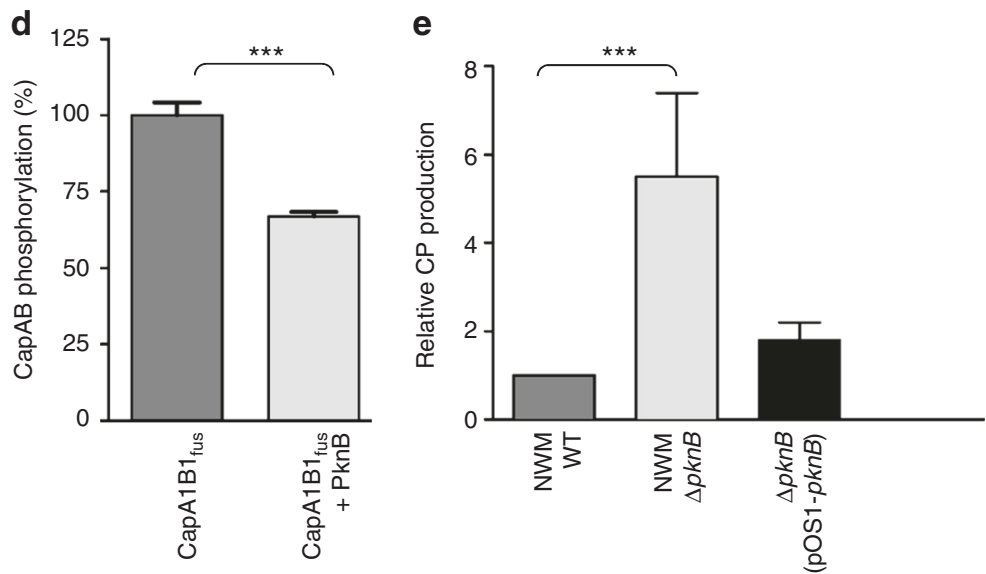

Fig. 6 CapM and CapB1 are protein targets of the ESTK PknB. a CapM is phosphorylated by PknB. Putative target proteins ( $3 \mu \mathrm{g})$ were incubated with PknB in the presence of [33P]ATP and reactions were analyzed by SDS-PAGE and phosphoimaging. $\mathbf{b}$ Impact of PknB kinase activity on CapM GT activity. CapM was incubated in presence of either active PknB (light grey) or heat-inactivated PknB (dark grey) with UDP-D- $\left[{ }^{14} \mathrm{C}\right]$ FucNAc and $\mathrm{C}_{55} \mathrm{P}$. After extraction with $\mathrm{BuOH} / \mathrm{Pyr} \mathrm{Ac}$, reactions were analyzed by TLC and phosphoimaging. Experiments were performed in triplicate. The error bars represent the \pm SD from three biological replicates. Statistical significance was analyzed by an unpaired $t$-test $\left({ }^{\star \star} p<0.05\right)$. c CapB1 is phosphorylated by PknB. Putative target proteins $(3 \mu \mathrm{g})$ were incubated with PknB in the presence of [ $\left.{ }^{33} \mathrm{P}\right]$ ATP, and reactions were analyzed by SDS-PAGE and phosphoimaging. $\mathbf{d}$ Quantification of $\mathrm{CapB}$ autophosphorylation in the absence and presence of PknB. Experiments were performed in triplicate. The error bars represent the \pm SD from three biological replicates. Statistical significance was analyzed by an unpaired $t$-test $\left({ }^{\star \star \star} p<0.005\right)$. e ELISA-based quantification of $C P$ production in a $\Delta p k n B$ deletion strain, the corresponding parental strain S. aureus Newman (WT) and complementation of the $\Delta p k n B$ mutant in trans. Experiments were performed in triplicate. The error bars represent the \pm standard error of the mean (SEM) from three biological replicates. Statistical significance was analyzed by an unpaired $t$-test $\left({ }^{\star \star \star} p<0.005\right)$

Reversible protein phosphorylation appears an elegant mechanism to ensure a coordinated and temporally controlled flux of intimately shared cell envelope metabolites. Several biosynthetic enzymes involved in polysaccharide production have been identified as endogenous substrates of bacterial tyrosine kinases, for instance the UDP-glucose 6-dehydrogenases TuaA and YwqF of Bacillus subtilis 54 , the homologous E. coli UDPglucose dehydrogenase $\mathrm{Ugd}^{55}$ and the UDP-ManNAc dehydrogenase $\mathrm{CapO}^{17}$ involved in S. aureus $\mathrm{CP}$ biosynthesis.

The fact that $\mathrm{CapO}$, involved in the third $\mathrm{CP}$ reaction cascade (Fig. 1), is a regulatory target of the CapAB kinase complex ${ }^{17}$, led us to assume that additional checkpoints within the biosynthetic pathway are also controlled by $\mathrm{CapAB}$, which turned out to be the case for CapE and CapM. Four tyrosine residues in the dehydrogenase CapE were identified as the specific phosphorylation sites, with Tyr76, Tyr290 and Tyr293 being widely conserved among homologous proteins from different pathogens. Interestingly, these residues are located in strategic functional regions of CapE: next to the cofactor binding site, in close proximity to the active site, and within a recently described mobile loop (Fig. 3f). Crystal structure analysis identified this mobile latch to connect two CapE protomers within the hexameric complex (trimer of dimers) and showed that the latch of one dimerization partner is associated with the substrate-binding domain of the contiguous CapE monomer, and vice versa, suggesting that this mobile loop is involved in regulating the access of the UDP-D-GlcNAc substrate to the active site ${ }^{56}$. Since contacts to Tyr290 and Tyr293 appear to be involved in the interaction, phosphorylation might induce conformational changes that facilitate access of UDP-D-GlcNAc to the active site, thereby increasing CapE enzymatic activity.

For the monotopic GT CapM, we identified the conserved tyrosine residue at position 157 as the CapA1B1 regulatory phosphorylation site which is in good agreement with the work of Minic et al. (2007) and supports the hypothesis that phosphorylation of the equivalent site in the $S$. thermophilus 
phosphogalactosyl-transferase EpsE results in activation in vivo ${ }^{57}$. A recent structural analysis showed, that the equivalent tyrosine residue in PglC is involved in a hydrogen-bonding network that establishes intramolecular interactions between helices $\mathrm{A}, \mathrm{F}$ and $\mathrm{G}^{37}$ (Supplementary Figure 3c), suggesting that the phosphorylated amino acid side chain may affect relative strengths of hydrogen bonds and critical interactions in CapM.

Current hypotheses suggest a multifaceted role for BY-kinases in bacterial exopolysaccharide production. BY-kinase signalling may not only regulate the catalytic activity of polysaccharide biosynthetic enzymes, but also ensure the correct cellular localization of protein targets ${ }^{58}$. Moreover, it has been suggested that BY-kinases may interact with the export and polymerization machinery to control the level of CP production and/or CP chain length ${ }^{52,59}$. However, the exact mechanism through which this control is exerted, as well as the molecular signal sensed, remains enigmatic so far.

The phosphodiesterase activity of CapA1 described here is in good agreement with previous studies demonstrating the release of CP and WTA into the culture supernatant in LCP deletion mutants of different species ${ }^{60-63}$. An $l c p C$ deletion mutant of $S$. aureus accumulated $\mathrm{CP}$ in the culture supernatant, indicating that LcpC is the key LCP enzyme for attachment of CP to the peptidoglycan in S. aureus $^{21}$.

This study provides biochemical proof that LcpC catalyzes the attachment of CP to PG. Importantly, CP lipid precursor cleavage and transfer of the phosphosugar moiety were found to be enhanced in the presence of CapA1, suggesting that the transmembrane activator cooperates with LcpC by forming an interaction complex, thereby modulating the attachment of $\mathrm{CP}$ to the cell wall precursor. In line with this interpretation, Toniolo et al. (2015) reported that the extracellular domain of the CapA1 homolog CpsC of Streptococcus agalactiae may modulate LCPmediated CP attachment in response to the phosphorylation state of the BY kinase $\mathrm{CpsD}^{64}$.

The natural PG acceptor substrate of LcpC is elusive; possible acceptor structures include the ultimate PG precursor lipid II, as well as "nascent" and crosslinked PG. More recently, LcpA and LcpB were shown to attach a shortened, soluble $\left(\mathrm{C}_{20} \mathrm{P}\right)$ WTA precursor to a preformed "nascent $\mathrm{PG}$ " oligomer in vitro ${ }^{65}$, but not to a lipid $\mathrm{II}_{\mathrm{PG}}$ mimetic lacking the natural undecaprenyl tail. In contrast, the biochemical analyses presented here clearly demonstrate that $\mathrm{CP}$ is efficiently attached to lipid $\mathrm{II}_{\mathrm{PG}}$ by the LcpC enzyme, and that PBPs are able to polymerize the resulting reaction product. With regard to the membrane localization of both, substrates and enzymes, and the interdependence and intimate connection of the enzymatic machineries, lipid $\mathrm{II}_{\mathrm{PG}}$ seems the most plausible acceptor.

CP lipid intermediates were further hydrolyzed by LcpC in the absence of the acceptor substrate as predicted by structural analysis of Cps2A of S. pneumoniae ${ }^{20}$, which was not observed for LcpA and LcpB proteins ${ }^{65}$. In vitro, all three CP lipid intermediates were efficiently processed by LcpC, although the proximal full-length undecaprenyl-pyrophosphoryl-linked sugar moiety appears sufficient for CP precursor recognition. Likewise, the first $\mathrm{C}_{55} \mathrm{P}$-linked sugar unit of the $\mathrm{O}$-antigen repeat unit contains the recognition information necessary for catalysis by the $\mathrm{O}$-antigen ligase $\mathrm{WaaL}^{66}$.

The biosyntheses of the cell envelope components PG, WTA and $\mathrm{CP}$ have to be coordinated in time and space, since the enzymatic machineries and their individual components are functionally related to each other or intimately connected ${ }^{1,53}$. The complex interplay between different cell envelope pathways becomes evident upon inhibition of individual biosynthetic steps. In analogy to the WTA biosynthesis in S. aureus, where late stage biosynthetic genes have been shown to be conditionally essential ("essential gene paradox";67), Yother and co-workers reported that deletion of the late stage CP biosynthesis genes, responsible for side chain assembly, polymerization or transport (cps $2 K$, cps $2 J$, and $\operatorname{cps} 2 \mathrm{H}$ ) in Streptococcus pneumoniae, is lethal ${ }^{68}$. Since the capsule is not required for cell viability per se, the damage to the cell envelope is most likely due to an inhibitory effect on PG biosynthesis, resulting from sequestration of CP lipid intermediates probably reducing the undecaprenyl-phosphate level to a critical point where PG synthesis is affected. Since we found CapA1 to hydrolyze CP lipid intermediates even in the absence of LCP proteins and that depletion of CapA1 in a $S$. aureus $\Delta l c p$ triple mutant was lethal, we conclude that CapA1-mediated cleavage of dead-end lipid-linked products might serve as a rescue mechanism counteracting depletion of $\mathrm{C}_{55} \mathrm{P}$ to critical levels.

The ESTK PknB is another important player in the orchestration of cell wall polymer biosynthesis and has been implicated in coordinating PG cross-wall formation, autolysis and cell division in $S$. aureus ${ }^{48-50}$. Our findings that $\mathrm{PknB}$ downregulates CapM and with the BY-kinase CapA1B1 operating in an antagonal manner underpins the importance of CapM as crucial enzymatic checkpoint. Moreover, in vitro kinase assays indicate that PknB-mediated phosphotransfer onto CapB1 modulates the activity of the CapA1B1 BY-kinase complex itself, thus identifying another activity by which $\mathrm{PknB}$ may exert control on CP biosynthesis. Cross-phosphorylation of ESTKs and BYkinases has also been reported in B. subtilis ${ }^{69}$. The CapB homo$\log$ PtkA was identified as in vitro phosphorylation target of the ESTK PrkC ${ }^{69}$. Moreover, PtkA autophosphorylation in vivo was strongly enhanced in a $\Delta p r k C$ strain, suggesting that PtkA BY-kinase activity is negatively regulated by PrkC-mediated phosphotransfer ${ }^{70}$

Corroborating the in vitro results, CP production was substantially elevated in a $p k n B$ deletion mutant of $S$. aureus. Besides decreasing the activity of CP biosynthesis proteins by direct phosphorylation, $\mathrm{PknB}$ kinase activity may serve to increase the cellular concentration of UDP-MurNAc-pentapeptide and lipid $\mathrm{II}_{\mathrm{PG}}{ }^{71}$, cell wall metabolites that were identified as inhibitors of CapD enzymatic activity ${ }^{19}$. Moreover, $\mathrm{PknB}$ signaling was reported to influence the transcription of the cap5 gene cluster ${ }^{72}$, suggesting that this sensor kinase is part of a multilayered signaltransduction network that coordinates $\mathrm{CP}$ formation in space and time.

The finding that $\mathrm{CP}$ and PG production are inversely controlled by PknB further substantiates a key role for PknB in the coordinate regulation of Gram-positive cell surface glycopolymers. In exponentially growing cells, PknB-mediated Ser/Thr phosphorylation positively controls PG biosynthesis, while exerting negative control on CP production (Fig. 7).

With respect to biosynthesis of the $S$. aureus CP, the CapA1B1 complex may be particularly important for short-term regulation. More recently, Elsholz and co-workers showed that exopolysaccharide (EPS) production in Bacillus subtilis is subject to a positive feedback loop that ties the synthesis of the polymer to its own concentration ${ }^{46}$. Regulation of EPS synthesis is mediated by the EpsAB tyrosine kinase complex, whereby direct recognition of EPS by the extracellular domain of the membrane component EpsA seems to result in stimulation of kinase activity ${ }^{46}$.

The activator/phosphodiesterase CapA1 directly and specifically interacts with lipid-bound CP precursors, and CapA1B1 signaling stimulates the activity of $\mathrm{CP}$ biosynthetic enzymes, triggering enhanced $\mathrm{CP}$ production in late-exponential and stationary phase (Fig. 7).

S. aureus $\mathrm{PknB}$ specifically interacts with lipid $\mathrm{II}_{\mathrm{PG}}$, likely responding to cellular pool levels of the ultimate peptidoglycan precursor $^{51}$. It is thus conceivable that in order to balance the pool levels of the lipid $\mathrm{II}_{\mathrm{PG}}$ acceptor and the $\mathrm{CP}$ donor substrate, 


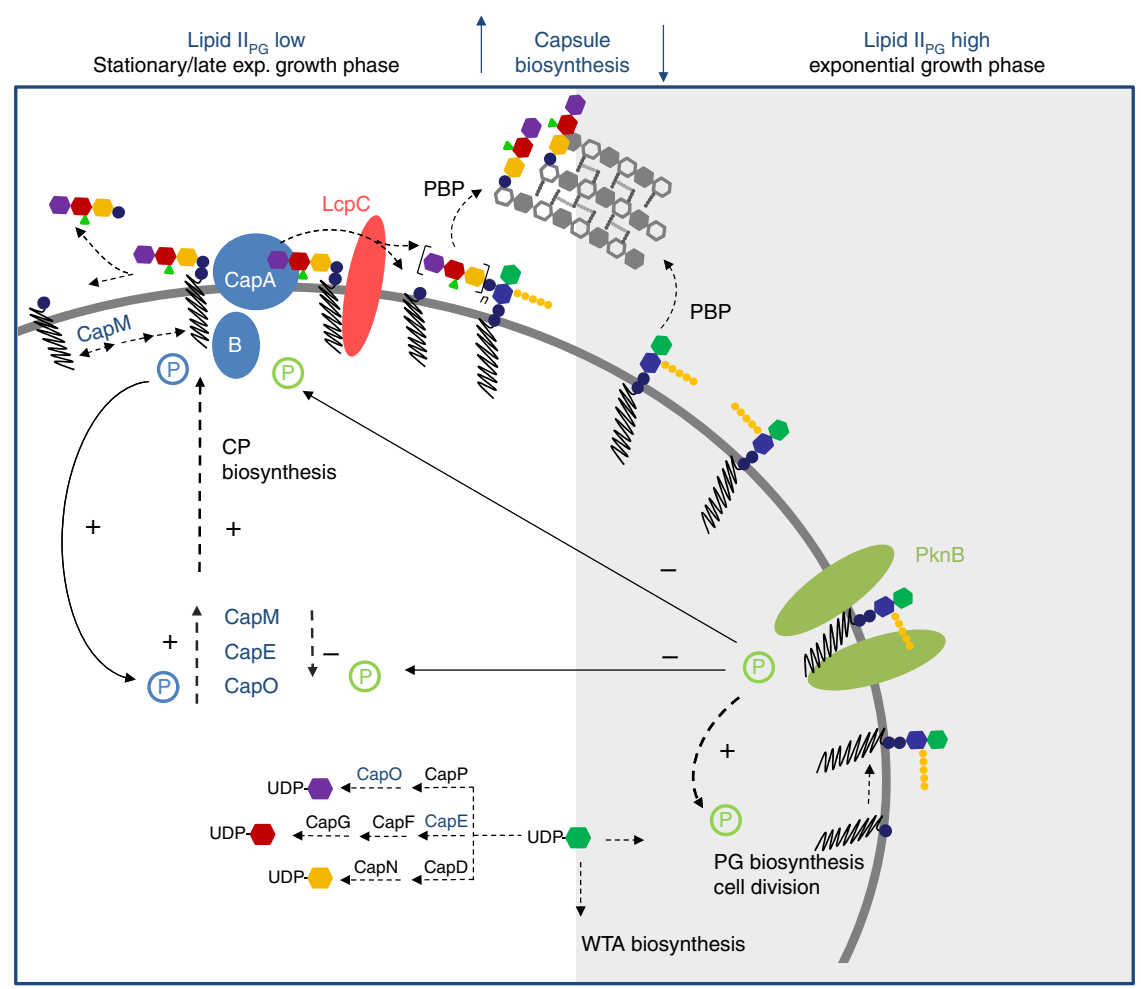

Fig. 7 Model for the regulation of CP biosynthesis and the attachment to PG in S. aureus. The CapA1B1 tyrosine complex (blue) positively controls multiple enzymatic checkpoints to orchestrate CP biosynthesis and precursor consumption during late exponential growth phase (white box). By catalyzing tyrosine phosphorylation (blue ' $\mathrm{P}$ ') of biosynthetic enzymes CapE, CapO and CapM, CapA1B1 positively controls the synthesis of both soluble and lipid-linked CP precursors. The membrane-anchored component CapA1 possesses a dual function; CapA1 recognizes lipid-bound CP building blocks and catalyzes the cleavage of the pyrophosphate linkage, releasing the essential isoprenoid lipid carrier $C_{55} P$. CP transfer is catalyzed by LcpC (red), which uses the ultimate peptidoglycan (PG) precursor lipid $\|_{P G}$ as an acceptor substrate. During exponential growth phase (grey shading), the insertion of new PG material (LII $P$ ) into the cell wall network is increased; UDP-GIcNAc and $\mathrm{C}_{55} \mathrm{P}$ are preferably channeled into PG and WTA biosynthesis, while CP production is low. The $\mathrm{Ser} / \mathrm{Thr}$ kinase PknB (green) contributes to the control of cell wall biosynthesis and cell division. Control of CP formation during ongoing PG synthesis is achieved by PknB via Ser/Thr phosphorylation (green ' $P$ '). PknB negatively controls the activity of the priming glycosyltransferase CapM to regulate the initial $\mathrm{C}_{55} \mathrm{P}$-consuming reaction within the $\mathrm{CP}$ biosynthetic pathway. Furthermore, $\mathrm{PknB}$ cross-talks with the CapAB complex, thus allowing control of the CP biosynthetic machinery on multiple levels, in response to the status of PG biosynthesis. The model highlights central biosynthetic precursors as important signal molecules situated at the interface of different pathways, integrated in feedback loops to control biosyntheses and the flux of shared precursors

which are both built on the lipid carrier $\mathrm{C}_{55} \mathrm{P}, \mathrm{PknB}$ and CapA1B1 antagonistically control biosyntheses to ensure vital cycling of the essential $\mathrm{C}_{55} \mathrm{P}$ lipid carrier. In addition, CapA1 might contribute to the correct localization of the CP assembly machinery, as proposed for S. agalactiae ${ }^{64}$, which has to integrate precursor transport and CP polymerization. This interaction protein network thus appears to contribute to the spatiotemporal coordination of CP biosynthesis with PG synthesis (Fig. 7), rendering proteins such as CapA1 and LcpC, exposed at the cell surface and devoid of eukaryotic homologs, potentially attractive antibacterial or antivirulence targets.

\section{Methods}

Strains. S. aureus Newman NCTC8178 was maintained on blood or lysogeny broth (LB; Oxoid) agar. Escherichia coli strains BL21 and C43 used for overexpression of recombinant $\mathrm{His}_{6}$-tag fusion proteins were maintained on LB-agar plates containing $50 \mu \mathrm{g} \mathrm{ml}^{-1}$ ampicillin or $25 \mu \mathrm{g} \mathrm{ml}^{-1}$ kanamycin. CP5 production was quantified from $S$. aureus cultivated on Columbia medium supplemented with $2 \% \mathrm{NaCl}$ and $1.5 \%$ agar. The Newman $\Delta p k n B$ mutant was kindly provided by Dr. Knut Olsen (Wuerzburg, Germany), and RN4220 carrying pCapA1 was provided by Dr. Gabriele Bierbaum (Bonn, Germany) ${ }^{44}$. S. aureus strain MW2 (NRS123) and strains from the Nebraska transposon mutant library ${ }^{73}$ were obtained from the Network on Antimicrobial Resistance in Staphylococcus aureus program, which was supported by the National Institute of Allergy and Infectious Diseases of the National Institutes of Health (contract HHSN272200700055C). The Nebraska library is comprised of derivatives of the serotype 5 strain USA300 LAC (cured of three plasmids) in which individual nonessential genes were disrupted by the insertion of the mariner transposon (Tn) bursa aurealis and includes mutants in capA2 (NE1286), capB2 (NE75), capA1 (NE302a), and capB1 (NE135). Construction of cap deletion mutants is described in the Supplementary Methods The $p k n B$ and Tn mutants were maintained on tryptic soy agar (TSA) plates with $5 \mu \mathrm{g} \mathrm{ml}^{-1}$ erythromycin, and pCapAl was maintained in $S$. aureus cultivated on TSA with $10 \mu \mathrm{g} \mathrm{ml}^{-1}$ chloramphenicol. S. aureus Newman $\triangle l c p A B C$ mutants were kindly provided by Dr. Olaf Schneewind (Chicago, USA) ${ }^{21}$ maintained on LB-agar plates. $S$. aureus strains carrying the plasmid pEPSA5 or the antisense plasmid pEPSA5-capA1AS were maintained on TSA-agar plates supplemented with $34 \mu \mathrm{g} \mathrm{ml}^{-1}$ chloramphenicol.

Plasmid construction and site-directed mutagenesis. Oligonucleotide primers were purchased from Eurofins MWG Operon (Germany). Primer pairs used for amplification of genomic sequences encoding capA1, capA2, capB1, capB2, capA1B1fus, capA2B2fus, capAlas, capC1, capC2, capI, capL, capN, capM and lcpC are listed in Supplementary Table 1. PCR reactions were carried out using Phusion DNA polymerase (NEB) and genomic DNA of the serotype 5 strain $S$. aureus Newman as template. PCR products were digested with type II restriction endonucleases (NEB; Supplementary Table 1) and ligated (T4 DNA ligase, Roche) with appropriately restricted expression vectors (Novagen). The PCR amplicon of the gene capN was inserted into pET24a. The vector pET21b was utilized for cloning of the capI and capL genes. The antisense fragment of capA1 was inserted into pEPSA5. All other amplicons were introduced into pET28a. The resulting plasmids (Supplementary Table 2) were confirmed by DNA sequencing (Sequiserve, Germany). Plasmids pET24a-capD, pKBK50d, pET5F1.1, pKBK6a, pET28a-pglF and pET52b-pknB used for overexpression of CapD-His ${ }_{6}$, CapE-His ${ }_{6}$, CapF-His 6 , CapG-His 6 , PglF-His ${ }_{6}$ and PknB-His ${ }_{6}$ have been described previously ${ }^{13,19}$. To 
enable expression of $\mathrm{N}$-terminally $\mathrm{His}_{6}$-tagged CapA1B1 and CapA2B2 fusion proteins, the constructs capA1B1fus and capA2B2fus were amplified using the primer pairs capA1_F and capB1_R, and capA2_F and $c a p B 2 \_R$, respectively. The QuikChange Lightning Mutagenesis Kit (Stratagene) was used according to the manufacturer's instructions to remove the stop codon of capA by site-directed mutagenesis (TTA $>\mathrm{GCA}$ ); the respective mutagenesis primers (capAlB1fus_mut_F, capA1B1fus_mut_R) and capA2B2fus_mut_F and capA2B2fus_mut_R (TAA $>$ TAT) are given in Supplementary Table 1. Site-directed mutagenesis of plasmid pET28a-capM was performed to introduce amino acid exchanges Tyr75 (TAT) > Phe (TTT) (primers: capM_mutY75_F, capM_mutY75_R) and Tyr157 (TAC) $>$ Phe (TTC) (capM_mutY157_F, capM_mutY157_R) in CapM. Likewise, plasmid pKBK50d was mutated to introduce amino acid exchanges Tyr76 (TAC) $>$ Phe (TTC) (capE_mutY76_F, capE_mutY76_R), Tyr290/293 (TAT) > Phe (TTT) (capE_mutY290/293_F, capE_mutY290/293_R), and Tyr329 (TAT) $>$ Phe (TTT) (capE_mutY329_F, capE_mutY329_R) into CapE. Site-directed mutagenesis of plasmid pET28a-capA1B1 was performed to introduce amino acid exchanges Thr8 (ACA) > Glu (GAA) (primers: capA1B1fus_mutT8E_F, capA1B1fus_mutT8E_R) and Thr8 (ACA) > Ala (GCA) (primers: capA1B1fus_mutT8A_F and capA1B1fus_mutT8A_R) into CapA1B1 fus. In order to produce a CapB2_T8E mutant, sitedirected mutagenesis of plasmid pET28a-capB2 was performed using the primer pair capB2_mutT8E_F and capB2_mutT8E_R to introduce an amino acid exchange Thr8 (ACA) > Glu (GAA). PCR-mediated base pair exchange was confirmed by sequencing. The resulting plasmids, which were utilized for expression and purification of CapM and CapE mutant proteins containing single or multiple Tyr to Phe exchanges, are listed in Supplementary Table 2. pCapA1 was transduced from S. aureus RN4220 to strain MW2 with phage 11, with selection on medium containing $10 \mu \mathrm{g} \mathrm{ml}^{-1}$ chloramphenicol. S. aureus Newman $\triangle l c p A B C$ was transformed by electroporation ${ }^{74}$ with pEPSA5-capA1AS purified from E. coli DC10B, with selection on TSA containing $2.5 \%$ glucose and $34 \mu \mathrm{g} \mathrm{ml}^{-1}$ chloramphenicol. Expression of the appropriate antisense fragment was induced on TSA-agar plates containing $500 \mathrm{mM}$ xylose.

Overexpression and purification of recombinant proteins. E. coli strain BL21 (DE3) (Promega) was used as host for the recombinant expression of cytoplasmic enzymes (CapB1-His 6 , CapB2-His 6 , CapC1-His 6 , CapC2-His 6 , CapE-His 6 , CapF$\mathrm{His}_{6}, \mathrm{CapG}-\mathrm{His}_{6}$, CapO-His ${ }_{6}, \mathrm{CapP}-\mathrm{His}_{6}$ and $\mathrm{PglF}-\mathrm{His}_{6}$ ) and E. coli strain $\mathrm{C} 43$ (DE3) (Promega) was used for the recombinant expression of integral membrane or membrane associated proteins (CapA1-His ${ }_{6}, \mathrm{CapA}_{2}-\mathrm{His}_{6}, \mathrm{CapAB}-\mathrm{His}_{6}$ fusion constructs, CapI-His ${ }_{6}, \mathrm{CapL}_{-} \mathrm{His}_{6}, \mathrm{CapM}-\mathrm{Hi}_{6}, \mathrm{CapD} \mathrm{His}_{6}, \mathrm{LcpC}^{\circ}-\mathrm{His}_{6}$ and Streptagged PknB. E. coli strain BL21 (DE3) cells were grown at $37^{\circ} \mathrm{C}$ in lysogeny broth (LB; Oxoid) and E. coli C43 (DE3) cells were grown at $30^{\circ} \mathrm{C}$ in double-strength yeast extract-tryptone broth (2YT, Difco) containing the appropriate selective antibiotic $\left(50 \mu \mathrm{g} \mathrm{ml}^{-1}\right.$ ampicillin or $25 \mu \mathrm{g} \mathrm{ml}^{-1}$ kanamycin). At an $\mathrm{OD}_{600}$ of 0.6 , IPTG was added at a final concentration of $0.5 \mathrm{mM}$ to induce expression of the recombinant protein. Expression times and temperatures were optimized for the individual constructs to ensure high yields of the different fusion proteins. The cultures for overexpression of CapA1, CapA2, CapAB fusion constructs, CapD, CapI, CapL, CapN, LcpC and PknB were induced at $20^{\circ} \mathrm{C}$ for $16 \mathrm{~h}$. All other E. coli BL21 (DE3) cultures and the E. coli C43 (DE3) cultures for the expression of CapM were induced at $30^{\circ} \mathrm{C}$ for $4 \mathrm{~h}$. After induction, cells were harvested $\left(15 \mathrm{~min}, 4^{\circ} \mathrm{C}\right.$, $7000 \times g)$ and resuspended in lysis buffer $(50 \mathrm{mM}$ Tris- $\mathrm{HCl}, 300 \mathrm{mM} \mathrm{NaCl}, \mathrm{pH}$ 7.5), which was supplemented with $1 \%(\mathrm{v} / \mathrm{v})$ Triton X-100 (Sigma-Aldrich) for purification of CapA1, CapA2, CapAB fusion constructs and CapN or with $29 \mathrm{mM}$ $n$-dodecyl- $\beta$-D-maltoside (DDM, Glycon Biochemicals) for purification of CapD, CapI, CapL, CapM and LcpC. Lysozyme $\left(250 \mu \mathrm{g} \mathrm{ml}^{-1}\right)$, DNase $\left(50 \mu \mathrm{g} \mathrm{ml}^{-1}\right)$ and RNase $\left(10 \mu \mathrm{g} \mathrm{ml}^{-1}\right)$ (Sigma-Aldrich) were added to the suspension; cells were incubated on ice for $30 \mathrm{~min}$ and sonicated. Cell debris was removed by centrifugation $\left(20 \mathrm{~min}, 4^{\circ} \mathrm{C}, 21,000 \times \mathrm{g}\right)$.

The supernatant was incubated with Ni-NTA-agarose slurry (Qiagen, Germany) for $2 \mathrm{~h}$ at $4{ }^{\circ} \mathrm{C}$ under gentle stirring. The mixture was then loaded onto a column support. After washing with lysis buffer, weakly bound material was removed with 10 and $20 \mathrm{mM}$ imidazole. Recombinant proteins were eluted with buffer containing $300 \mathrm{mM}$ imidazole. Five $500-\mu \mathrm{l}$ fractions were collected each and stored in $30 \%$ (v/v) glycerol at $-20^{\circ} \mathrm{C}$; preparations of CapE were dialyzed against $10 \mathrm{mM}$ potassium phosphate buffer (KPi), $\mathrm{pH} 7.5$ before storage. For PknB purification the pellet was resuspended in lysis buffer containing $1 \%(\mathrm{v} / \mathrm{v})$ Triton $\mathrm{X}-100$ and the suspension was incubated on ice for $1 \mathrm{~h}$. Cell debris was spun down ( $20 \mathrm{~min}, 4^{\circ} \mathrm{C}, 34,000 \mathrm{x} \mathrm{g}$ ) and the clear supernatant was loaded onto a Strep-tactin agarose column (IBA, Germany). The column was washed twice with lysis buffer and PknB was eluted with Strep-tactin elution buffer (IBA, Germany). Purity of elution fractions was assessed by SDS-Page (NuPAGE; Invitrogen); protein concentrations were measured using Bradford reagent (Bio-Rad, Germany). Expression and purification of CapA $1 \mathrm{Bl}_{\text {fus }}, \mathrm{CapE}$ and $\mathrm{CapM}$ protein variants were carried out as described for the wild-type proteins.

In vitro syntheses of soluble CP precursors. UDP-D-GlcNAc and cofactors for enzyme assays were obtained from Sigma-Aldrich. Synthesis of the precursor UDP-2-acetamido-2,6-dideoxy-D-xylo-4-hexulose for mass spectrometric analysis was performed with a recombinant truncated version of the enzyme PglF from C. jejuni, as previously described ${ }^{19}$. The PglF reaction product was used as substrate for reconstitution of CapN catalytic activity. For this purpose, PglF reactions were carried out overnight and quenched by heating $\left(5 \mathrm{~min}, 95^{\circ} \mathrm{C}\right)$. CapN catalyzed synthesis of UDP-D-FucNAc was carried out in a total volume of $40 \mu \mathrm{l}$. CapN $(12 \mu \mathrm{g})$ was incubated in the presence of $\sim 3 \mathrm{mM}$ UDP-2-acetamido2,6-dideoxy-D-xylo-4-hexulose, $1.875 \mathrm{mM} \mathrm{NADPH,} 8 \%(\mathrm{v} / \mathrm{v})$ Triton X-100 and $10 \mathrm{mM} \mathrm{KPi}, \mathrm{pH} 7.5$, for $2 \mathrm{~h}$ at $30^{\circ} \mathrm{C}$. Synthesis of larger quantities of soluble capsule precursors for MALDI-TOF MS analysis was achieved by a 10 -fold upscale of the synthesis reaction.

Alternatively, synthesis of UDP-D-FucNAc was performed in an one-potassay containing $4 \mu \mathrm{g}$ CapD, $4 \mu \mathrm{g}$ CapN, $2 \mathrm{mM}$ UDP-D-GlcNAc, $2 \mathrm{mM}$ NADPH and $0.5 \mathrm{mM} \mathrm{NADP}$ in $50 \mathrm{mM}$ Tris- $\mathrm{HCl}, 10 \mathrm{mM} \mathrm{MgCl} 2, \mathrm{pH} 7.5$, in a total volume of $30 \mu \mathrm{l}$. UDP-L-FucNAc was synthesized by incubating CapE, CapF and CapG in presence of $2 \mathrm{mM}$ UDP-D-GlcNAc, $2 \mathrm{mM}$ NADPH and $0.5 \mathrm{mM}$ NADP in $50 \mathrm{mM}$ Tris-HCl, $10 \mathrm{mM} \mathrm{MgCl}_{2}$, pH 7.5 .

The third soluble precursor UDP-D-ManNAcA was synthesized by incubating $2 \mathrm{mM}$ UDP-D-GlcNAc with CapO and CapP ( $4 \mu \mathrm{g}$ each), $6 \mathrm{mM} \mathrm{NAD}^{+}$and 0.5 $\mathrm{mM}$ dithiothreitol (DTT; Sigma-Aldrich) in $50 \mathrm{mM}$ Tris- $\mathrm{HCl}, 10 \mathrm{mM} \mathrm{MgCl} 2, \mathrm{pH}$ 7.5. All enzymatic synthesis reactions were quenched by heating $5 \mathrm{~min}, 90^{\circ} \mathrm{C}$ ).

In vitro modulation of CapE catalytic activity by CapAB. The influence of CapAB-mediated tyrosine phosphorylation on CapE catalytic activity was examined in vitro using purified recombinant proteins. Assays were performed in a total volume of $100 \mu \mathrm{l}$ containing $17 \mu \mathrm{g}$ of CapE (or of a CapE protein variants) and $8.5 \mu \mathrm{g}$ of purified CapAB kinase complex. The enzymes were incubated in the presence of $3 \mathrm{mM}$ UDP-D-GlcNAc and $1 \mathrm{mM} \mathrm{ATP}$, in $10 \mathrm{mM} \mathrm{KPi}, 10 \mathrm{mM} \mathrm{MgCl}_{2}$, pH 7.5 supplemented with $1 \mathrm{mM} \mathrm{DTT}$, for $30 \mathrm{~min}$ at $30^{\circ} \mathrm{C}$. Reactions were quenched by heating $\left(5 \mathrm{~min}, 90^{\circ} \mathrm{C}\right)$, then subjected to capillary electrophoresis (CE) analysis ${ }^{19}$. Control reactions were performed with heat-inactivated $(10 \mathrm{~min}$, $\left.100^{\circ} \mathrm{C}\right)$ CapAB protein.

MALDI-TOF mass spectrometric analysis. Soluble capsule precursors were purified by RP18-HPLC ${ }^{19}$, and cleaved $\mathrm{C}_{55} \mathrm{P}$ intermediates were purified by preparative TLC. Lipid spots were visualized using iodine vapour and extracted off the silica plates with methanol. Samples were spotted onto a ground steel MALDITOF target plate and allowed to dry at room temperature. Subsequently, each sample was overlaid with $1 \mu \mathrm{l}$ of matrix (saturated solution of 6-Aza-2-thiothymine in $50 \%(\mathrm{v} / \mathrm{v})$ ethanol, $10 \mathrm{mM}$ diammonium hydrogen citrate) and air dried at room temperature again. Spectra were recorded either in the reflector negative mode within a mass range from 300 to $3000 \mathrm{Da}$ (soluble capsule precursors) or in the linear negative mode within a mass range from 400 to $3000 \mathrm{Da}$ (lipid intermediates), at a laser frequency of $9 \mathrm{~Hz}$ on a Biflex III mass spectrometer (Bruker Daltonics). Data analysis was performed using flexAnalysis software (Bruker Daltonics)

In vitro kinase assays. In vitro BY-kinase assays were carried out in a total volume of $10 \mu \mathrm{l}$ containing either $0.5 \mu \mathrm{g}$ of CapA and CapB, or $2 \mu \mathrm{g}$ of a CapAB protein fusion construct. For identification of protein substrates, $2 \mu \mathrm{g}$ of a recombinant target protein were added. The proteins were incubated in the presence of $10 \mu \mathrm{Ci} \gamma$-labeled $\left.{ }^{33} \mathrm{P}\right] \mathrm{ATP}(\sim 300 \mathrm{nM}$; Hartmann Analytic) in $50 \mathrm{mM}$ Tris- $\mathrm{HCl}, 10 \mathrm{mM} \mathrm{MgCl}$, $\mathrm{pH} 7.5$ supplemented with $0.5 \mathrm{mM}$ DTT, $0.5 \mathrm{mM}$ EDTA and $10 \mu \mathrm{M}$ ATP. Assays with CapAB protein variants were carried out as described for the wild-type proteins.

For identification of PknB protein substrates, purified PknB $(0.5 \mu \mathrm{g})$ was incubated in presence of $3 \mathrm{mM} \mathrm{MnCl} 2$ in the reaction mixture described above. Cross-phosphorylation of CapAB by PknB was assessed analogously.

After $30 \mathrm{~min}$ of incubation at $30^{\circ} \mathrm{C}$, reactions were stopped by addition of $4 \mathrm{x}$ LDS sample buffer (Invitrogen), and analyzed by SDS-PAGE (NuPAGE, Invitrogen). Radioactive protein bands were visualized using a storage phosphor screen in a Storm imaging system (GE Healthcare). A detailed description of the identification of phosphorylation sites by nanoLC-MS/MS is given in the Supplementary Methods.

In vitro phosphatase assays. CapC phosphatase activity was examined in vitro by the addition of CapC1 or CapC2 $\left(2 \mu \mathrm{g}\right.$ each) with $2 \mathrm{mM} \mathrm{MnCl}_{2}$ to the CapAB in vitro kinase assays (see above) and subsequent heat-inactivation. After incubation for $1 \mathrm{~h}$ at $30^{\circ} \mathrm{C}$, phosphatase reaction was stopped by the by addition of 4x LDS sample buffer (Invitrogen), and analyzed by SDS-PAGE (NuPAGE, Invitrogen). Radioactive protein bands were visualized using a storage phosphor screen in a Storm imaging system (GE Healthcare).

In vitro syntheses of lipid $\mathrm{I}_{\text {capr }}$ lipid $\mathrm{II}_{\text {capr }}$ and lipid $\mathrm{III}_{\text {cap. }}$. Lipid $\mathrm{I}_{\text {cap }}$ synthesis was carried out in a total volume of $50 \mu \mathrm{l}$ containing $5 \mathrm{nmol} \mathrm{C}_{55} \mathrm{P}$ (Larodan, Sweden), $2 \mathrm{mM}$ UDP-D-GlcNAc, $10 \mathrm{mM}$ NADPH, $0.5 \mathrm{mM}$ NADP and $0.6 \%(\mathrm{v} / \mathrm{v})$ DMSO in $50 \mathrm{mM}$ Tris- $\mathrm{HCl}, 10 \mathrm{mM} \mathrm{MgCl}, \mathrm{pH} 7.5$. The reaction was initiated by the addition of $4 \mu \mathrm{g}$ of biosynthetic enzymes CapD, CapN and CapM, and incubated for $16 \mathrm{~h}$ at $30^{\circ} \mathrm{C}$.

To assess the influence of different detergents and phospholipids on CapM activity, newly synthesized UDP-D-FucNAc (described above) was incubated with $4 \mu \mathrm{g} \mathrm{CapM}, 5 \mathrm{nmol} \mathrm{C}_{55} \mathrm{P}$ in a reaction mixture containing $50 \mathrm{mM}$ Tris- $\mathrm{HCl}, 10 \mathrm{mM}$ 
$\mathrm{MgCl}_{2}, \mathrm{pH} 7.5$ supplemented with an appropriate amount of triton X-100, $n$-dodecyl- $\beta$-D-maltoside (DDM), $n$-lauroyl sarcosine (LS), dimethylsulfoxide (DMSO), dioleoylphosphatidylglycerol (DOPG) or cardiolipin (CL). To investigate the modulatory effect of CapAB on CapM activity $4 \mu \mathrm{g}$ CapAB and $10 \mathrm{mM}$ ATP were added to the CapM reaction mixture. To assess the influence of $\mathrm{PknB}$ on CapM activity in vitro, $1 \mu \mathrm{g}$ PknB was added to the CapM reaction mixture supplemented with $2 \mathrm{mM} \mathrm{MnCl}$ and $10 \mathrm{mM}$ ATP.

The second precursor lipid $\mathrm{II}_{\text {cap }}$ was synthesized in a total volume of $50 \mu \mathrm{l}$ by incubating the capsule biosynthesis proteins CapE, CapF, CapG and CapL $(4 \mu \mathrm{g}$ each) in the presence of $2 \mathrm{nmol}$ purified lipid $\mathrm{I}_{\text {cap }}$ and $2 \mathrm{mM}$ UDP-D-GlcNAc, 2 $\mathrm{mM}$ NADPH, $0.5 \mathrm{mM}$ NADP and $0.6 \%$ (v/v) DMSO in $50 \mathrm{mM}$ Tris-HCl, $10 \mathrm{mM}$ $\mathrm{MgCl}_{2}$, pH 7.5.

Lipid III $_{\text {cap }}$ was synthesized by incubating $2 \mathrm{nmol}$ purified lipid II $_{\text {cap }}$ with CapO, CapP and CapI ( $4 \mu \mathrm{g}$ each) and $2 \mathrm{mM}$ UDP-D-GlcNAc, $6 \mathrm{mM} \mathrm{NAD}^{+}, 0.5 \mathrm{mM}$ dithiothreitol (DTT; Sigma-Aldrich) in $50 \mathrm{mM}$ Tris- $\mathrm{HCl}, 10 \mathrm{mM} \mathrm{MgCl}_{2}, \mathrm{pH} 7.5$. Synthesized CP precursors were extracted from the reaction mixture with an equal volume of $n$-butanol/pyridine acetate, pH $4.2(2: 1, \mathrm{v} / \mathrm{v})$ and analyzed by TLC on silica plates (Merck) according to Rick (chloroform, methanol, water, ammonium hydroxide, $88: 48: 10: 1)^{75}$. Nisin was added at a molar ratio of 2:1 with respect to the lipid precursor prior to the extraction procedure. Reaction mixtures were extracted and unbound lipids were analyzed by TLC. For mass spectrometric analysis of lipid intermediates see Supplementary Methods.

For synthesis of $\left[{ }^{14} \mathrm{C}\right]$-labeled lipid-bound precursors, the assays were further supplemented with $0.333 \mathrm{nmol}$ UDP-D- $\left[{ }^{14} \mathrm{C}\right] \mathrm{GlcNAc}$ (Hartmann Analytic, Germany). Radiolabeled spots were visualized using a storage phosphor screen in a Storm imaging system (GE Healthcare). Non-radiolabeled lipid intermediates were analyzed using PMA staining reagent $(2.5 \%(\mathrm{w} / \mathrm{v})$ phosphomolybdate, $1 \%(\mathrm{w} / \mathrm{v})$ ceric sulfate, $6 \%(\mathrm{v} / \mathrm{v})$ sulfuric acid). Isolation of small quantities of CP precursors was achieved by synthesis and subsequent purification via preparative TLC. To this end, lipid spots were visualized using iodine vapour and material was scratched off the silica plates. Lipids were extracted by incubation in $100 \mu \mathrm{l}$ of methanol for 60 min. Larger quantities of $\mathrm{CP}$ precursor were purified using high-performance liquid chromatography (HPLC) over a DEAE-FF ( $5 \mathrm{ml}$; GE Healthcare) and eluted in a linear gradient from chloroform/ methanol/ water (2:3:1) to chloroform, methanol, $300 \mathrm{mM}$ ammonium bicarbonate (2:3:1).

CapA1 catalyzed cleavage of lipid-bound CP precursor. For reconstitution of the CapA1 mediated cleavage of the pyrophosphate-linkage of lipid-linked cell wall intermediates, purified CapA1 $(8 \mu \mathrm{g})$ was incubated with $2 \mathrm{nmol}$ lipid $\mathrm{I}_{\text {cap }}$, lipid $\mathrm{III}_{\mathrm{WTA}}$, lipid $\mathrm{II}_{\mathrm{PG}}$ in $10 \mathrm{mM} \mathrm{MgCl}$ and $50 \mathrm{mM}$ Tris- $\mathrm{HCl}, \mathrm{pH}$ 7.5. After incubation for $16 \mathrm{~h}$ at $30^{\circ} \mathrm{C}$, cleavage products were extracted from the reaction mixture with an equal volume of $n$-butanol/pyridine acetate, $\mathrm{pH} 4.2(2: 1, \mathrm{v} / \mathrm{v})$ and analyzed by TLC on silica plates (Merck) according to Rick (chloroform, methanol, water, ammonium hydroxide, 88:48:10:1) and visualized by PMA staining ${ }^{75}$.

In vitro LCP assays. For reconstitution of the $\mathrm{CP}$ ligation reaction purified lipid $\mathrm{I}_{\text {cap }}$ or $\left[{ }^{14} \mathrm{C}\right]$ lipid $\mathrm{I}_{\text {cap }}$ was incubated in the presence of LcpC $(4 \mu \mathrm{g})$, using $2 \mathrm{nmol}$ of purified lipid $\mathrm{II}_{\mathrm{PG}}$ or $\left[{ }^{14} \mathrm{C}\right]$ lipid $\mathrm{II}_{\mathrm{PG}}$ as acceptor substrate in a $50 \mu$ reaction mixture with $0.6 \%$ DMSO, $18 \mu \mathrm{g}$ DOPG, $10 \mathrm{mM} \mathrm{MgCl}{ }_{2}$ in $50 \mathrm{mM}$ MES buffer, $\mathrm{pH}$ 5.5. Synthesis and purification of the acceptor substrate lipid II is described in the supplementary methods. If indicated, assays additionally contained $4 \mu \mathrm{g}$ of CapA1. After incubation for $16 \mathrm{~h}$ at $30^{\circ} \mathrm{C}$, samples were analyzed by TLC, followed by phosphoimaging. Polymerization of LcpC reaction products were catalyzed by the subsequent addition of PBP2 with $2 \mathrm{mM} \mathrm{CaCl}_{2}$ to the LCP reaction mixture, followed by incubation at $30^{\circ} \mathrm{C}$ for $1 \mathrm{~h}$. Moenomycin (MOE) was used to block the PBP2 catalyzed reaction and added at a final concentration of $10 \mu \mathrm{M}$. For evaluation of LcpC mediated hydrolysis of CP precursor in the absence of the acceptor substrate, $2 \mathrm{nmol}$ of the individual lipid-linked precursor was incubated with $4 \mu \mathrm{g}$ LcpC, $18 \mu \mathrm{g}$ DOPG, $10 \mathrm{mM} \mathrm{MgCl}_{2}$ and 0.6\% DMSO in $50 \mathrm{mM}$ MES buffer, pH 5.5. After incubation for $16 \mathrm{~h}$ at $30^{\circ} \mathrm{C}$, samples were analyzed by TLC and visualized by PMA staining.

Reporting summary. Further information on experimental design is available in the Nature Research Reporting Summary linked to this article.

\section{Data availability}

The authors declare that all data supporting the findings of this study are available within the Article and its Supplementary Information.

Received: 21 March 2016 Accepted: 28 February 2019

Published online: 29 March 2019

\section{References}

1. Hanson, B. R. \& Neely, M. N. Coordinate regulation of Gram-positive cell surface components. Curr. Opin. Microbiol. 15, 204-210 (2012).
2. Bouhss, A., Trunkfield, A. E., Bugg, T. D. H. \& Mengin-Lecreulx, D. The biosynthesis of peptidoglycan lipid-linked intermediates. FEMS Microbiol. Rev. 32, 208-233 (2008)

3. Xia, G., Kohler, T. \& Peschel, A. The wall teichoic acid and lipoteichoic acid polymers of Staphylococcus aureus. Int. J. Med. Microbiol. 300, 148-154 (2010).

4. David, M. Z. \& Daum, R. S. Community-Associated Methicillin-Resistant Staphylococcus aureus: Epidemiology and Clinical Consequences of an Emerging Epidemic. Clin. Microbiol. Rev. 23, 616-687 (2010).

5. Thakker, M., Park, J.-S., Carey, V. \& Lee, J. C. Staphylococcus aureus serotype 5 capsular polysaccharide is antiphagocytic and enhances bacterial virulence in a murine bacteremia model. Infect. Immun. 66, 5183-5189 (1998).

6. Nilsson, I. M., Lee, J. C., Bremell, T., Rydén, C. \& Tarkowski, A. The role of staphylococcal polysaccharide microcapsule expression in septicemia and septic arthritis. Infect. Immun. 65, 4216-4221 (1997).

7. Portolés, M., Kiser, K. B., Bhasin, N., Chan, K. H. N. \& Lee, J. C. Staphylococcus aureus cap5o has udp-mannac dehydrogenase activity and is essential for capsule expression. Infect. Immun. 69, 917-923 (2001).

8. Boyle-Vavra, S. et al. USA300 and USA500 clonal lineages of staphylococcus aureus do not produce a capsular polysaccharide due to conserved mutations in the cap5 locus. mBio6, e0258-14 (2015).

9. Diekema, D. J. et al. Continued emergence of USA300 methicillin-resistant staphylococcus aureus in the united states: results from a nationwide surveillance study. Infect. Control. Hosp. Epidemiol. 35, 285-292 (2014).

10. van der Mee-Marquet, N. et al. The incidence of Staphylococcus aureus ST8 USA300 among French pediatric inpatients is rising. Eur. J. Clin. Microbiol. Infect. Dis. 34, 935-942 (2015).

11. Jones, C. Revised structures for the capsular polysaccharides from Staphylococcus aureus Types 5 and 8, components of novel glycoconjugate vaccines. Carbohydr. Res. 340, 1097-1106 (2005).

12. Sau, S. et al. The Staphylococcus aureus allelic genetic loci for serotype 5 and 8 capsule expression contain the type-specific genes flanked by common genes. Microbiology 143, 2395-2405 (1997).

13. Kneidinger, B. et al. Three highly conserved proteins catalyze the conversion of UDP-n-acetyl-d-glucosamine to precursors for the biosynthesis of $\mathrm{O}$ antigen in Pseudomonas aeruginosa $\mathrm{O} 11$ and capsule in staphylococcus aureus type 5 implications for the udp-n-acetyl-l-fucosamine biosynthetic pathway. J. Biol. Chem. 278, 3615-3627 (2003).

14. Kiser, K. B., Bhasin, N., Deng, L. \& Lee, J. C. Staphylococcus aureus cap5P encodes a UDP-N-acetylglucosamine 2-epimerase with functional redundancy. J. Bacteriol. 181, 4818-4824 (1999).

15. Bhasin, N. et al. Identification of a gene essential for O-acetylation of the Staphylococcus aureus type 5 capsular polysaccharide. Mol. Microbiol. 27, 9-21 (1998)

16. Soulat, D. et al. Staphylococcus aureus operates protein-tyrosine phosphorylation through a specific mechanism. J. Biol. Chem. 281, 14048-14056 (2006).

17. Soulat, D., Grangeasse, C., Vaganay, E., Cozzone, A. J. \& Duclos, B. UDPacetyl-mannosamine dehydrogenase is an endogenous protein substrate of Staphylococcus aureus protein-tyrosine kinase activity. J. Mol. Microbiol. Biotechnol. 13, 45-54 (2007).

18. O'Riordan, K. \& Lee, J. C. Staphylococcus aureus capsular polysaccharides Clin. Microbiol. Rev. 17, 218-234 (2004).

19. Li, W. et al. Analysis of the Staphylococcus aureus capsule biosynthesis pathway in vitro: Characterization of the UDP-GlcNAc C6 dehydratases CapD and CapE and identification of enzyme inhibitors. Int. J. Med. Microbiol. IJMM 304, 958-969 (2014).

20. Kawai, Y. et al. A widespread family of bacterial cell wall assembly proteins. EMBO J. 30, 4931-4941 (2011).

21. Chan, Y. G.-Y., Kim, H. K., Schneewind, O. \& Missiakas, D. The capsular polysaccharide of Staphylococcus aureus is attached to peptidoglycan by the LytR-CpsA-Psr (LCP) family of enzymes. J. Biol. Chem. 289, 15680-15690 (2014).

22. Barreteau, H. et al. Quantitative high-performance liquid chromatography analysis of the pool levels of undecaprenyl phosphate and its derivatives in bacterial membranes. J. Chromatogr. B 877, 213-220 (2009).

23. Swoboda, J. G. et al. Discovery of a small molecule that blocks wall teichoic acid biosynthesis in Staphylococcus aureus. ACS Chem. Biol. 4, 875-883 (2009).

24. D’Elia, M. A., Millar, K. E., Beveridge, T. J. \& Brown, E. D. Wall teichoic acid polymers are dispensable for cell viability in Bacillus subtilis. J. Bacteriol. 188, 8313-8316 (2006)

25. Sewell, E. W. \& Brown, E. D. Taking aim at wall teichoic acid synthesis: new biology and new leads for antibiotics. J. Antibiot. (Tokyo) 67, 43-51 (2014).

26. Pohlmann-Dietze, P. et al. Adherence of Staphylococcus aureus to endothelial cells: influence of capsular polysaccharide, global regulator agr, and bacterial growth Phase. Infect. Immun. 68, 4865-4871 (2000). 
27. Wamel, Wvan et al. Regulation of Staphylococcus aureus type 5 capsular polysaccharides by agr and sarA in vitro and in an experimental endocarditis model. Microb. Pathog. 33, 73-79 (2002).

28. Luong, T. T. \& Lee, C. Y. The arl locus positively regulates Staphylococcus aureus type 5 capsule via an mgrA-dependent pathway. Microbiology 152, 3123-3131 (2006).

29. Grangeasse, C., Nessler, S. \& Mijakovic, I. Bacterial tyrosine kinases: evolution, biological function and structural insights. Philos. Trans. R. Soc. B 367, 2640-2655 (2012).

30. Lee, D. C. \& Jia, Z. Emerging structural insights into bacterial tyrosine kinases. Trends Biochem. Sci. 34, 351-357 (2009).

31. Grangeasse, C., Cozzone, A. J., Deutscher, J. \& Mijakovic, I. Tyrosine phosphorylation: an emerging regulatory device of bacterial physiology. Trends Biochem. Sci. 32, 86-94 (2007).

32. Bender, M. H., Cartee, R. T. \& Yother, J. Positive Correlation between tyrosine phosphorylation of $\mathrm{CpsD}$ and capsular polysaccharide production in Streptococcus pneumoniae. J. Bacteriol. 185, 6057-6066 (2003).

33. Morona, J. K., Paton, J. C., Miller, D. C. \& Morona, R. Tyrosine phosphorylation of $\mathrm{CpsD}$ negatively regulates capsular polysaccharide biosynthesis in Streptococcus pneumoniae. Mol. Microbiol. 35, 1431-1442 (2000).

34. Müller, A., Ulm, H., Reder-Christ, K., Sahl, H.-G. \& Schneider, T. Interaction of type A lantibiotics with undecaprenol-bound cell envelope precursors. Microb. Drug. Resist. 18, 261-270 (2012).

35. Breton, C., Snajdrová, L., Jeanneau, C., Koca, J. \& Imberty, A. Structures and mechanisms of glycosyltransferases. Glycobiology 16, 29R-37R (2006).

36. Patel, K. B., Ciepichal, E., Swiezewska, E. \& Valvano, M. A. The C-terminal domain of the Salmonella enterica WbaP (UDP-galactose: Und-P galactose-1phosphate transferase) is sufficient for catalytic activity and specificity for undecaprenyl monophosphate. Glycobiology 22, 116-122 (2012).

37. Ray, L. C. et al. Membrane association of monotopic phosphoglycosyl transferase underpins function. Nat. Chem. Biol. 14, 538-541 (2018).

38. Gasteiger, E. et al. in The Proteomics Protocols Handbook (ed. Walker, J.) 571-607 (Humana Press, Totowa, NJ, 2005).

39. Price, N. P. \& Momany, F. A. Modeling bacterial UDP-HexNAc: polyprenol-P HexNAc-1-P transferases. Glycobiology 15, 29R-42R (2005).

40. Walvoort, M. T. C., Lukose, V. \& Imperiali, B. A modular approach to phosphoglycosyltransferase inhibitors inspired by nucleoside antibiotics. Chem. - Eur. J. 22, 3856-3864 (2016).

41. Gruszczyk, J. et al. Comparative analysis of the Tyr-kinases CapB1 and CapB2 fused to their cognate modulators CapA1 and CapA2 from Staphylococcus aureus. PLOS ONE 8, e75958 (2013).

42. Blom, N., Sicheritz-Pontén, T., Gupta, R., Gammeltoft, S. \& Brunak, S. Prediction of post-translational glycosylation and phosphorylation of proteins from the amino acid sequence. Proteomics 4, 1633-1649 (2004).

43. Kavanagh, K. L., Jörnvall, H., Persson, B. \& Oppermann, U. Medium-and short-chain dehydrogenase/reductase gene and protein families. Cell. Mol. Life Sci. 65, 3895-3906 (2008).

44. Jansen, A. et al. Production of capsular polysaccharide does not influence Staphylococcus aureus vancomycin susceptibility. Bmc. Microbiol. 13, 65 (2013).

45. Montgomery, C. P. et al. Comparison of virulence in community-associated methicillin-resistant Staphylococcus aureus pulsotypes USA300 and USA400 in a rat model of pneumonia. J. Infect. Dis. 198, 561-570 (2008).

46. Elsholz, A. K., Wacker, S. A. \& Losick, R. Self-regulation of exopolysaccharide production in Bacillus subtilis by a tyrosine kinase. Genes Dev. 28, 1710-1720 (2014).

47. Eberhardt, A. et al. Attachment of capsular polysaccharide to the cell wall in Streptococcus pneumoniae. Microb. Drug. Resist. 18, 240-255 (2012).

48. Donat, S. et al. Transcriptome and functional analysis of the eukaryotic-type serine/threonine kinase PknB in Staphylococcus aureus. J. Bacteriol. 191, 4056-4069 (2009).

49. Beltramini, A. M., Mukhopadhyay, C. D. \& Pancholi, V. Modulation of cell wall structure and antimicrobial susceptibility by a Staphylococcus aureus eukaryote-like serine/threonine kinase and phosphatase. Infect. Immun. 77, 1406-1416 (2009).

50. Tamber, S., Schwartzman, J. \& Cheung, A. L. Role of PknB kinase in antibiotic resistance and virulence in community-acquired methicillin-resistant Staphylococcus aureus Strain USA300. Infect. Immun. 78, 3637-3646 (2010).

51. Hardt, P. et al. The cell wall precursor lipid II acts as a molecular signal for the Ser/Thr kinase PknB of Staphylococcus aureus. Int. J. Med. Microbiol. 307, 1-10 (2017).

52. Olivares-Illana, V. et al. Structural basis for the regulation mechanism of the tyrosine kinase CapB from Staphylococcus aureus. PLoS Biol. 6, e143 (2008).
53. Yother, J. Capsules of Streptococcus pneumoniae and other bacteria: paradigms for polysaccharide biosynthesis and regulation. Annu. Rev. Microbiol. 65, 563-581 (2011).

54. Mijakovic, I. et al. Transmembrane modulator-dependent bacterial tyrosine kinase activates UDP-glucose dehydrogenases. EMBO J. 22, 4709-4718 (2003).

55. Grangeasse, C. et al. Autophosphorylation of the Escherichia coli protein kinase Wzc regulates tyrosine phosphorylation of Ugd, a UDP-glucose dehydrogenase. J. Biol. Chem. 278, 39323-39329 (2003).

56. Miyafusa, T., Caaveiro, J. M. M., Tanaka, Y., Tanner, M. E. \& Tsumoto, K. Crystal structure of the capsular polysaccharide synthesizing protein CapE of Staphylococcus aureus. Biosci. Rep. 33, 463-474 (2013).

57. Minic, Z. et al. Control of EpsE, the phosphoglycosyltransferase initiating exopolysaccharide synthesis in Streptococcus thermophilus, by EpsD tyrosine kinase. J. Bacteriol. 189, 1351-1357 (2007).

58. Jers, C. et al. Bacillus subtilis BY-kinase PtkA controls enzyme activity and localization of its protein substrates. Mol. Microbiol. 77, 287-299 (2010).

59. Whitfield, C. \& Larue, K. Stop and go: regulation of chain length in the biosynthesis of bacterial polysaccharides. Nat. Struct. Mol. Biol. 15, 121-123 (2008).

60. Massidda, O., Kariyama, R., Daneo-Moore, L. \& Shockman, G. D. Evidence that the PBP 5 synthesis repressor (psr) of Enterococcus hirae is also involved in the regulation of cell wall composition and other cell wall-related properties. J. Bacteriol. 178, 5272-5278 (1996).

61. Cieslewicz, M. J., Kasper, D. L., Wang, Y. \& Wessels, M. R. Functional analysis in type ia group b streptococcus of a cluster of genes involved in extracellular polysaccharide production by diverse species of streptococci. J. Biol. Chem. 276, 139-146 (2001).

62. Morona, J. K., Morona, R. \& Paton, J. C. Attachment of capsular polysaccharide to the cell wall of Streptococcus pneumoniae type 2 is required for invasive disease. Proc. Natl. Acad. Sci. 103, 8505-8510 (2006).

63. Hübscher, J. et al. MsrR contributes to cell surface characteristics and virulence in Staphylococcus aureus. FEMS Microbiol. Lett. 295, 251-260 (2009).

64. Toniolo, C. et al. Streptococcus agalactiae capsule polymer length and attachment is determined by the proteins CpsABCD. J. Biol. Chem. 290, 9521-9532 (2015)

65. Schaefer, K., Matano, L. M., Qiao, Y., Kahne, D. \& Walker, S. In vitro reconstitution demonstrates the cell wall ligase activity of LCP proteins. Nat. Chem. Biol. 13, 396-401 (2017).

66. Horzempa, J., Dean, C. R., Goldberg, J. B. \& Castric, P. Pseudomonas aeruginosa 1244 pilin glycosylation: glycan substrate recognition. J. Bacteriol. 188, 4244-4252 (2006).

67. D'Elia, M. A. et al. Lesions in teichoic acid biosynthesis in Staphylococcus aureus lead to a lethal gain of function in the otherwise dispensable pathway. J. Bacteriol. 188, 4183-4189 (2006).

68. Xayarath, B. \& Yother, J. Mutations blocking side chain assembly, polymerization, or transport of a Wzy-dependent Streptococcus pneumoniae capsule are lethal in the absence of suppressor mutations and can affect polymer transfer to the cell wall. J. Bacteriol. 189, 3369-3381 (2007).

69. Shi, L. et al. Cross-phosphorylation of bacterial serine/threonine and tyrosine protein kinases on key regulatory residues. Front. Microbiol. 5, 495 (2014).

70. Ravikumar, V. et al. Quantitative phosphoproteome analysis of Bacillus subtilis reveals novel substrates of the kinase PrkC and phosphatase PrpC. Mol. Cell. Proteom. MCP 13, 1965-1978 (2014).

71. Liebeke, M., Meyer, H., Donat, S., Ohlsen, K. \& Lalk, M. A Metabolomic view of Staphylococcus aureus and Its Ser/Thr kinase and phosphatase deletion mutants: involvement in cell wall biosynthesis. Chem. Biol. 17, 820-830 (2010).

72. Burnside, K. et al. Regulation of hemolysin expression and virulence of Staphylococcus aureus by a serine/threonine kinase and phosphatase. PLoS ONE 5, el1071 (2010).

73. Fey, P. D. et al. A genetic resource for rapid and comprehensive phenotype screening of nonessential Staphylococcus aureus genes. mBio 4, e00537-00512 (2013).

74. Monk, I. R., Shah, I. M., Xu, M., Tan, M.-W. \& Foster, T. J. Transforming the untransformable: application of direct transformation to manipulate genetically Staphylococcus aureus and Staphylococcus epidermidis. mBio 3, e00277 (2012)

75. Rick, P. D. et al. Characterization of the lipid-carrier involved in the synthesis of enterobacterial common antigen (ECA) and identification of a novel phosphoglyceride in a mutant of Salmonella typhimurium defective in ECA synthesis. Glycobiology 8, 557-567 (1998).

\section{Acknowledgements}

We would like to thank Michaele Josten for mass spectrometry, and Katie O'Riordan for providing plasmids pKBK4, pKBK6a, pKBK10, pKBK50d, pET24-capD, pET24-capN, 
and pET5F1.1. We thank Jerry Pier for providing purified PNAG, Knut Ohlsen for providing strain $S$. aureus Newman $\Delta p k n B$, Gabriele Bierbaum for providing plasmid pCapA1, Andreas Peschel for providing E. coli DC10 $\beta$ and Olaf Schneewind for kindly providing the $\triangle l c p A B C$ mutant. Marvin Rausch is grateful for support by the Juergen Manchot Foundation. This work was supported by the German Research Foundation (DFG; SCHN 1284/1-2) and the BONFOR program of the Medical Faculty of the University of Bonn.

\section{Author contributions}

M.R., J.P.D., H.U., A.M., W.L., P.H., M.S., M.E., X.W., X.L. performed experiments. C.E. M., W.V., H.G.S., J.C.L., T.S. contributed to the study design, planning of the experiments and interpretation of data. H.U. and T.S. wrote the manuscript. M.R., A.M., C.E. M., W.V., J.C.L., H.G.S., T.S. contributed to revision of the manuscript.

\section{Additional information}

Supplementary Information accompanies this paper at https://doi.org/10.1038/s41467019-09356-x.

Competing interests: The authors declare no competing interests.

Reprints and permission information is available online at http://npg.nature.com/ reprintsandpermissions/

Journal peer review information: Nature Communications thanks Eric Brown and the other anonymous reviewer(s) for their contribution to the peer review of this work. Peer reviewer reports are available.

Publisher's note: Springer Nature remains neutral with regard to jurisdictional claims in published maps and institutional affiliations.

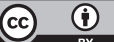

adap appropriate credit to the original author(s) and the source, provide a link to the Creative material in this article are included in the article's Creative Commons license, unless indicated otherwise in a credit line to the material. If material is not included in the article's Creative Commons license and your intended use is not permitted by statutory regulation or exceeds the permitted use, you will need to obtain permission directly from the copyright holder. To view a copy of this license, visit http://creativecommons.org/ licenses/by/4.0/.

(C) The Author(s) 2019 